\title{
Effects of Antibiotic Withdrawal on Multidrug Resistant Escherichia coli
}

\author{
Hitesh Kumar* and Sunil Maherchandani \\ Department of veterinary Microbiology, CVAS, Bikaner (Raj.), India \\ *Corresponding author
}

\section{A B S T R A C T}

\begin{tabular}{|l|}
\hline K e y w o r d s \\
Multidrug-resistance, \\
$\begin{array}{l}\text { Efflux activity, Efflux } \\
\text { pumps, Antibiotic } \\
\text { withdrawal }\end{array}$ \\
\hline Article Info \\
\hline $\begin{array}{l}\text { Accepted: } \\
\text { 04 September } 2018 \\
\text { Available Online: } \\
\text { 10 October } 2018\end{array}$ \\
\hline
\end{tabular}

Antimicrobial resistance (AMR) is an increasingly serious threat to global public health. The increase in antibiotic consumption is well evident which accelerates the AMR. The continuous dissemination of 'multidrug-resistant' (MDR) bacteria drastically reduces the efficacy of antibiotics and consequently increases the frequency of therapeutic failure. In MDR bacteria the over-expression of efflux pumps that expels antibiotics which contribute to the increased resistance by decreasing the intracellular concentration of antibiotics. In the present study 42 MDR isolates from different groups (human, pig, and poultry) were evaluated for their antibiogram profile, mechanism of antibiotic resistance prevalent in them and to know the effects of withdrawal of antibiotics on bacterial resistance MDR bacteria were sub-culture. Withdrawal of antibiotic definitely helps in reverting the resistant phenotype in majority of the MDRs. The recent investigation on the AMR, including adaptive mechanism to resistance for various antibiotics, may pave the way towards the decreased frequency of emergence of Escherichia coli strains that are highly resistant.

\section{Introduction}

Antibiotic resistance mechanisms reported in Gram-negative bacteria are causing a worldwide health problem. AMR develops when a microorganism no longer responds to a drug to which it was originally sensitive. This AMR occurs naturally over time. However, the misuse and overuse of antimicrobials accelerates this process. The increase in antibiotic consumption is well evident from the fact that there has been an increase of antibiotic usage by $30 \%$ globally between 2000 and 2010 (Van Boeckel et al., 2014). In India itself a $40 \%$ increase in the use of antibiotics has been reported in a survey from
2005 to 2009 (Ganguly et al., 2011). The burden of increased antibiotic use is reflected in the fact that infectious disease mortality rate in India currently is 416.75 per 100,000 persons (Laxminarayan and Chaudhury, 2016).

Reductions in antimicrobials use have been reported to achieve decrease in antimicrobial resistance (Barbosa and Levy, 2000; Nys et al., 2005).

AMR being a global burden of complex epidemiology, an integrated One Health approach is required to control this problem. One Health approach recognizes that the 
health of people is connected to the health of animals and the environment and AMR has clear links to each of these three domains. Resistant organisms exist in humans, animals, food and the environment and antibiotic resistant bacteria can be transmitted between the three domains (Robinson et al., 2016).

Antibiotic resistance in microbes can either be inherited/acquired or non-inherited/adaptive. Acquired antibiotic resistance is generally associated with extrachromosomal elements, like plasmids, transposons and integrons carrying antibiotic resistance genes, transferred from one bacteria to another through horizontal gene transfer or vertically from one generation of bacteria to the next (Courvalin, 1994).

Conversely, adaptive antibiotic resistance describes a form of transient resistance that occurs due to the organism's ability to adapt and phenotypically modify itself in order to develop resistance to certain stressors (Barclay and Begg, 2001), where pre-exposure to antibiotics remains a major cause of resistance. Adaptive mechanisms include expression of efflux pumps, reduced number of porins and other cell surface modifications created by stress of low level antibiotics. Adaptive resistance is ephemeral type of resistance and bacteria tend to come to its original state once the stressor is removed. However, a longer pre-exposure to subinhibitory levels of antibiotics can result in the emergence of permanent mutations.

\section{Materials and Methods}

\section{Sampling}

A total of 69 samples were collected from different groups' viz. human (32 urine samples), pig (20 faecal samples) and poultry (17 faecal samples).

Isolation and identification of bacterial

\section{strains}

After overnight enrichment of samples, cultures were streaked on to MacConkey agar. The plates of MacConkey agar (MCA) were incubated at $37^{\circ} \mathrm{C}$ for 24 hours. The pink lactose fermenting colonies were further streaked on to Eosin Methylene Blue (EMB) agar and incubated at $37^{\circ} \mathrm{C}$ for 24 hours.

The bacterial isolates showing characteristic metallic sheen were assigned laboratory numbers accordingly and confirmation of the isolates as Escherichia coli was done using Gram's staining, Catalase test, Oxidase test and a set of 25 biochemical tests provided in Hi 25 Enterobacteriaceae identification kit.

\section{Antibiotic sensitivity test}

The isolates were inoculated in sterile $5 \mathrm{ml}$ nutrient broth, incubated for $18 \mathrm{hr}$ at $37^{\circ} \mathrm{C}$ and then optical density was adjusted to 0.5 McFarland with Normal saline solution (Quinn et al., 1994).

Each inoculum was then spread over the surface of Mueller-Hinton agar with the help of sterile swab. Plates were allowed to dry for $10 \mathrm{~min}$ at $37^{\circ} \mathrm{C}$ and then antibiotic discs were carefully placed on the surface with enough space around each disc for diffusion of the antibiotic.

Plates were incubated for $24 \mathrm{hr}$ at $37^{\circ} \mathrm{C}$ and the zone of inhibition of growth of the organism around each disc was measured in millimeters. The antibiotic discs used for the experiment are given in table 1 .

\section{Ethidium bromide-agar (Et-br) cartwheel method for evaluation of MDR strains}

The test was performed as per the method of Martins et al., 2013.

Principle 
This is a subjective screening test in which it is assumed that the smallest concentration of Et-br that produces fluorescence of the bacterial mass represents the highest concentration of Et-br that the bacteria can exclude.

The extent of exclusion is based on either active efflux pumps which will pump out the ethidium bromide or reduction in the number of porins thereby preventing the entry of ethidium bromide.

\section{Ethidium Bromide (Et-br) agar Cartwheel} method

All isolates were grown in $5 \mathrm{ml}$ of nutrient broth and adjusted to $0.5 \mathrm{McFarland}$ with PBS.

Agar plates containing Et-br concentrations ranging from 0.5 to $2.5 \mathrm{mg} / \mathrm{l}$ were prepared on the same day of the experiment and protected from light by covering with aluminium foil.

The plates were then divided into as many as 8 sectors by radial lines (cartwheel pattern).

Cultures were swabbed on Et-br agar plates starting from the centre of the plate and spreading towards the edges.

The swabbed Et-br agar plates were then incubated at $37^{\circ} \mathrm{C}$ for $16 \mathrm{~h}$ and examined under UV transilluminator and photographed.

The minimum concentration of Et-br that produced fluorescence was recorded.

Evaluation of MDR $E$. coli isolates for adaptive resistance

All the isolates were sub-cultured for five times in nutrient broth. After each subculturing antibiotic sensitivity test and
Ethidium bromide-agar (Et-br) cartwheel test were performed.

\section{Results and Discussion}

A total of 42 MDR E. coli isolate from humans, pigs and poultry were studied.

\section{Antibiogram study for Escherichia coli}

In the present investigation, all $42 \mathrm{E}$. coli isolates were subjected to resistotyping with 29 different antibiotics (Table 1). The response of organisms was interpreted as sensitive, intermediate and resistant based on the CLSI guidelines. The percentage of resistance of all the isolates to a particular antibiotic is given in Table 2 .

The antibiotics tested belonged to various groups i.e. $\quad \beta$-lactam antibiotics, aminoglycosides, glycopeptides, phenicoles, quinolones, Tetracyclines, sulphonamide, RNA synthesis inhibitor, polypeptides, macrolides and lincosamides. $\beta$-lactam antibiotics included penicillins, cephalosporins, monobactums and carbapenems.

In $\beta$-lactum group (cell wall sysnthesis inhibitors) all the isolates were resistant to Penicillin-G and Oxacillin.

Other antibiotics studied in this group were representatives of all the four generations of Cephalosporins, Ampicillin, Ticarcillin, Pipracillin, Polymyxin-B, Aztreonam, Meropenam and Vancomycin. Of the above studied antibiotics there was comparatively more resistance observed for first $(95.2 \%)$ and second generation $(90.5 \%)$ cephalosporins and vancomycin $(97.6 \%)$. Human isolates had more resistance $(95 \%$ and $70 \%)$ for third and fourth generation cephalosporins i.e. Ceftazidime and Cefepime also, as compared to pig and poultry isolates. Pig isolates were 
least resistant with $58.3 \%$ and $16.7 \%$ resistivity to third and fourth generation cephalosporins respectively. On the contrary $60 \%$ and $50 \%$ poultry isolates were resistant to third and fourth generation cephalosporins respectively. Similarly, human and poultry isolates were more resistant to Ampicillin, Ticarcillin and Pipracillin (65\%, 65\%, 70\% and $60 \%, 40 \%, 60 \%$ respectively) as compare to pig isolates $(33.3 \%, 0 \%$ and $8.3 \%$ respectively). The resistivity patterns observed could be because of the difference in the antibiotic use of the hosts from which $E$. coli had been isolated. Since, history of the antibiotics being used in different hosts could not be collected no conclusive correlation could be obtained. Vancomycin has never been reported to be effective against $E$. coli as these Gram negative organisms have an intact outer membrane, which serves as a permeability barrier that blocks access of the antibiotic to the PG-D-Ala-D-Ala termini in the periplasm (Walsh et al., 1996). There were $60 \%$ of $E$. coli isolates of human and poultry origin resistant to Meropenam and 25\% isolates of pigs resistant to this antibiotic. Increased resistance to Meropenam is a point of concern as carbapenems are considered to be the ultimate drugs for the treatment of untreatable infections. Reasons for the resistance to these antibiotics could be the presence of active efflux, reduction in the number of porins or due to the activity of carbapenamases (Little et al., 2012).

Four antibiotics of aminoglycoside group were taken for the present study namely: Streptomycin, Gentamicin, Kanamycin and Amikacin. Isolates of $E$. coli from three species taken for the study showed variable resistivity patterns to all the antibiotics of these four groups. Isolates of human and pig origin were sensitive to Streptomycin as compared to poultry isolates. But, for Gentamicin, all the poultry isolates were sensitive where as $45 \%$ and $25 \%$ resistance was observed for human and pig isolates respectively. In case of Kanamycin high level of resistance was observed for human and poultry isolates (90\% and $80 \%$ respectively). $95 \%$ of human isolates were resistant to Amikacin but only $41.7 \%$ and $40 \%$ pig and poultry isolates respectively were resistant.

In general, five mechanisms of resistance have been reported for this class of antibiotics: (i) decreased intracellular accumulation of the antibiotic by alteration of outer membrane permeability, diminished inner membrane transport or active efflux (ii) enzymatic modification of the drug, which is the most common (iii) modification of the target by mutation in ribosomal proteins or $16 \mathrm{~S}$ rRNA genes (iv) trapping of the drug and (v) post transcriptional methylation of rRNA, using Sadenosyl-methionine as a cofactor (Magnet and Blanchard, 2005). Significant difference in resistivity observed for different antibiotics in relation to the species from which they were isolated indicate that this could be the affect of difference in the usage of the antibiotic and adaptive resistance rather than any other mechanism.

Human and pig isolates showed lower level of resistance to Tetracycline (30\% and $8.3 \%$ ) and Doxycycline $(20 \%$ and $0 \%)$ as compared to poultry isolates (90\% and 80\%). Higher antibiotic resistivity of $E$. coli isolates from poultry to tetracyclins was also observed earlier in this region (Meena, 2015). Significant difference in resistivity pattern of human and pig E. coli isolates from poultry isolates clearly indicate Tertacyclin abuse in the poultry. The most common mechanism for resistance to the Tetracyclines is energydependent drug efflux. This energy-dependent drug efflux resistance can be transient, or by chromosomal mutations resulting in over expression of existing chromosomal genes mexA, mexB, oprM and mar (Table 3 and 4). 
Table.1 List of antibiotics used for antibiogram study against $E$. coli

\section{Cell wall synthesis inhibitor}

\begin{tabular}{|l|}
\hline Class of antibiotics \\
\hline $1^{\text {st }}$ gen. Cephalosporin \\
\hline $2^{\text {nd }}$ gen. Cephalosporin \\
\hline $3^{\text {rd }}$ gen.cephaloantipseudo \\
\hline $3^{\text {rd }}$ gen. Cephalosporin \\
\hline $4^{\text {th }}$ gen. Cephalosporin \\
\hline Natural penicillin \\
\hline Penicillinase stable \\
\hline Aminopenicillin \\
\hline Carboxypenicillin \\
\hline Ureidopenicillin \\
\hline Polypeptide \\
\hline Monobactums \\
\hline Carbapenems \\
\hline Glycopeptides \\
\hline
\end{tabular}

\begin{tabular}{|l|l|}
\hline Antibiotics & Concentration (mcg) \\
\hline Cefazolin(CZ) & 30 \\
\hline Cefuroxime(CXM) & 30 \\
\hline Ceftazidime(CAZ) & 30 \\
\hline Cefotaxime(CTX) & 30 \\
\hline Cefipime(CPM) & 30 \\
\hline Penicillin-G(P) & 10 \\
\hline Oxacillin(OX) & 1 \\
\hline Ampicillin(AMP) & 10 \\
\hline Ticarcillin(TI) & 10 \\
\hline Pipracillin(PI) & 100 \\
\hline Polymyxin-B(PB) & 300 \\
\hline Aztreonam(AT) & 30 \\
\hline Meropenam(MRP) & 10 \\
\hline Vancomycin(VA) & 30 \\
\hline
\end{tabular}

Protein synthesis inhibitor (30-S)

\begin{tabular}{|l|l|l|}
\hline Aminoglycoside & Kanamycin(K) & 30 \\
\hline Aminoglycoside & Gentamicin(GEN) & 10 \\
\hline Aminoglycoside & Amikacin(Ak) & 30 \\
\hline Aminoglycoside & Streptomycin(S) & 10 \\
\hline Natural tetracycline & Tetracycline(TE) & 30 \\
\hline Semisynthetic tet. & Doxycycline(DO) & 30 \\
\hline
\end{tabular}

Protein synthesis inhibitor (50-S)

\begin{tabular}{|l|l|l|}
\hline Lincosamides & Clindamycin(CD) & 2 \\
\hline Macrolides(50-S) & Erythromycin(E) & 15 \\
\hline Phenicoles(50-S) & Chloramphenicol(C) & 30 \\
\hline DNA synthesis inhibitor & Nalidixic acid(NA) & 30 \\
\hline $\mathbf{1}^{\text {st }}$ gen quinolone & Ciprofloxacin(CIP) & 5 \\
\hline $\mathbf{2}^{\text {nd }}$ gen quinolone & Levofloxacin(LE) & 5 \\
\hline $\mathbf{3}^{\text {rd }}$ gen quinolone & Rifampicin(RIF) & 5 \\
\hline RNA synthesis inhibitor & \\
\hline RNA synthesis inhibitor & Trimethoprim(TR) & 5 \\
\hline Anti metabolite antibiotics (folic acid synthesis inhibitors) & \\
\hline Sulphonamide & Cotrimoxazole(COT) & 25 \\
\hline $\begin{array}{l}\text { Combination of sulpha } \\
\text { trimethoprim }\end{array}$ &
\end{tabular}


Table.2 Percent resistivity of all the 42 isolates of Escherichia coli to a given antibiotic

\begin{tabular}{|c|c|c|c|c|c|c|c|c|}
\hline Mechanism & Class of antibiotics & Antibiotics & $\begin{array}{c}\text { Sensitive } \\
(\%)\end{array}$ & $\begin{array}{c}\text { Intermediate } \\
(\%)\end{array}$ & $\begin{array}{l}\text { Total } \\
\text { Resistant } \\
\text { E. Coli } \\
(\%)\end{array}$ & $\begin{array}{c}\text { Resistant } \\
\text { E. coli } \\
\text { from } \\
\text { Human } \\
(\%)\end{array}$ & $\begin{array}{l}\text { Resistant } \\
\text { E. coli } \\
\text { from Pig } \\
(\%)\end{array}$ & $\begin{array}{c}\text { Resistant } \\
\text { E. coli } \\
\text { from } \\
\text { Poultry } \\
(\%)\end{array}$ \\
\hline \multirow{14}{*}{$\begin{array}{l}\text { Cell wall } \\
\text { Synthesis } \\
\text { Inhibitor }\end{array}$} & $1^{\text {st }}$ gen. Cephalosporin & Cefazolin & 2.4 & 2.4 & 95.2 & 100 & 91.6 & 90 \\
\hline & $2^{\text {nd }}$ gen. Cephalosporin & Cefuroxime & 2.4 & 7.1 & 90.5 & 80 & 100 & 100 \\
\hline & $3^{\text {rd }}$ gen.cephaloantipseudo & Ceftazidime & 16.7 & 7.1 & 76.2 & 95 & 58.3 & 60 \\
\hline & $3^{\text {rd }}$ gen. Cephalosporin & Cefotaxime & 11.9 & 21.4 & 66.7 & 85 & 58.3 & 40 \\
\hline & $4^{\text {th }}$ gen. Cephalosporin & Cefepime & 38.1 & 11.9 & 50 & 70 & 16.7 & 50 \\
\hline & Natural penicillin & Penicillin-G & 0 & 0 & 100 & 100 & 100 & 100 \\
\hline & Penicillinase stable & Oxacillin & 0 & 0 & 100 & 100 & 100 & 100 \\
\hline & Aminopenicillin & Ampicillin & 33.3 & 11.9 & 54.8 & 65 & 33.3 & 60 \\
\hline & Carboxypenicillin & Ticarcillin & 47.6 & 11.9 & 40.5 & 65 & $\mathbf{0}$ & 40 \\
\hline & Ureidopenicillin & Pipracillin & 26.2 & 23.8 & 50 & 70 & 8.3 & 60 \\
\hline & Polypeptide & Polymyxin-B & 50 & 0 & 50 & 40 & 50 & 70 \\
\hline & Monobactums & Aztreonam & 35.7 & 26.2 & 38.1 & 65 & 8.3 & 20 \\
\hline & Carbapenems & Meropenam & 31 & 19 & 50 & 60 & 25 & 60 \\
\hline & Glycopeptides & Vancomycin & 0 & 2.4 & 97.6 & 95 & 100 & 100 \\
\hline \multirow{6}{*}{$\begin{array}{l}\text { Protein } \\
\text { Synthesis } \\
\text { Inhibitor } \\
(30-S)\end{array}$} & Aminoglycoside & Kanamycin & 0 & 26.2 & 73.8 & 90 & 41.7 & 80 \\
\hline & Aminoglycoside & Gentamicin & 54.8 & 16.7 & 28.6 & 45 & 25 & $\mathbf{0}$ \\
\hline & Aminoglycoside & Amikacin & 19 & 14.3 & 66.7 & 95 & 41.7 & 40 \\
\hline & Aminoglycoside & Streptomycin & 40.5 & 40.5 & 19 & 15 & 8.3 & 40 \\
\hline & Natural tetracycline & Tetracycline & 54.8 & 7.1 & 38.1 & 30 & 8.3 & 90 \\
\hline & Semisynthetic tetracycline & Doxycycline & 61.9 & 9.5 & 28.6 & 20 & $\mathbf{0}$ & 80 \\
\hline \multirow{3}{*}{$\begin{array}{l}\text { Protein } \\
\text { Synthesis } \\
\text { inhibitor }(\mathbf{5 0 - S )}\end{array}$} & Lincosamides & Clindamycin & 0 & 0 & 100 & 100 & 100 & 100 \\
\hline & Macrolides & Erythromycin & 0 & 4.8 & 95.2 & 90 & 100 & 100 \\
\hline & Phenicoles & Chloramphenicol & 78.6 & 9.5 & 11.9 & 15 & 0 & 20 \\
\hline \multirow{3}{*}{$\begin{array}{l}\text { DNA synthesis } \\
\text { Inhibitor }\end{array}$} & $1^{\text {st }}$ gen quinolone & Nalidixic acid & 21.4 & 16.7 & 61.9 & 55 & 58.3 & 80 \\
\hline & $2^{\text {nd }}$ gen quinolone & Ciprofloxacin & 47.6 & 19 & 33.3 & 55 & $\mathbf{0}$ & 30 \\
\hline & $3^{\text {rd }}$ gen quinolone & Levofloxacin & 59.5 & 14.3 & 26.2 & 50 & $\mathbf{0}$ & 10 \\
\hline RNA inhibitor & RNA synthesis inhibitor & Rifampicin & 0 & 0 & 100 & 100 & 100 & 100 \\
\hline \multirow[t]{2}{*}{ Antimetabolite } & Sulphonamide & Trimethoprim & 54.8 & 2.4 & 42.9 & 65 & $\mathbf{0}$ & 50 \\
\hline & Sulpha + trimethoprim & Cotrimoxazole & 54.8 & 0 & 45.2 & 65 & 0 & 60 \\
\hline
\end{tabular}


Table.3 Antibiogram of different isolates of Escherichia coli from human

\begin{tabular}{|c|c|c|c|c|c|c|c|c|c|c|c|c|c|c|c|c|c|c|c|c|c|}
\hline Class of antibiotics & Antibiotics & $\begin{array}{c}\mathrm{HU} \\
2\end{array}$ & $\begin{array}{c}\mathrm{HU} \\
3\end{array}$ & $\begin{array}{c}\mathrm{HU} \\
4\end{array}$ & $\begin{array}{c}\mathrm{HU} \\
7\end{array}$ & $\begin{array}{r}\text { HS } \\
10\end{array}$ & $\begin{array}{c}\mathrm{HU} \\
12\end{array}$ & $\begin{array}{c}\mathrm{HU} \\
13\end{array}$ & $\begin{array}{r}\text { HU } \\
14\end{array}$ & $\begin{array}{c}\text { HU } \\
15\end{array}$ & $\begin{array}{c}\text { HU } \\
17\end{array}$ & $\begin{array}{c}\text { HU } \\
18\end{array}$ & $\begin{array}{c}\mathrm{HU} \\
20\end{array}$ & $\begin{array}{c}\text { HU } \\
22\end{array}$ & $\begin{array}{c}\mathrm{HU} \\
23\end{array}$ & $\begin{array}{c}\mathrm{HU} \\
24\end{array}$ & $\begin{array}{l}\text { HU } \\
27\end{array}$ & $\begin{array}{c}\text { HU } \\
29\end{array}$ & $\begin{array}{c}\text { HU } \\
30\end{array}$ & E.H & E. \\
\hline $1^{\text {st }}$ gen. Cephalosporin & Cefazolin & $\mathrm{R}$ & $\mathrm{R}$ & $\mathrm{R}$ & $\mathrm{R}$ & $\mathrm{R}$ & $\mathrm{R}$ & $\mathrm{R}$ & $\mathrm{R}$ & $\mathrm{R}$ & $\mathrm{R}$ & $\mathrm{R}$ & $\mathrm{R}$ & $\mathrm{R}$ & $\mathrm{R}$ & $\mathrm{R}$ & $\mathrm{R}$ & $\mathrm{R}$ & $\mathrm{R}$ & $\mathrm{R}$ & $\mathrm{R}$ \\
\hline $2^{\text {nd }}$ gen. Cephalosporin & Cefuroxime & $\mathrm{S}$ & $\mathrm{R}$ & $\mathrm{R}$ & $\mathrm{R}$ & $\mathrm{R}$ & I & $\mathrm{R}$ & I & I & $\mathrm{R}$ & $\mathrm{R}$ & $\mathrm{R}$ & $\mathrm{R}$ & $\mathrm{R}$ & $\mathrm{R}$ & $\mathrm{R}$ & $\mathrm{R}$ & $\mathrm{R}$ & $\mathrm{R}$ & $\mathrm{R}$ \\
\hline $3^{\text {rd }}$ gen. Cephalosporin & Cefotaxime & $\mathrm{R}$ & $\mathrm{R}$ & I & $\mathrm{R}$ & $\mathrm{R}$ & $\mathrm{R}$ & $\mathrm{R}$ & $I^{* *}$ & I & $\mathrm{R}$ & $\mathrm{R}$ & $\mathrm{R}$ & $\mathrm{R}$ & $\mathrm{R}$ & $\mathrm{R}$ & $\mathrm{R}$ & $\mathrm{R}$ & $\mathrm{R}$ & $\mathrm{R}$ & $\mathrm{R}$ \\
\hline $4^{\text {th }}$ gen. Cephalosporin & Cefipime & $\mathrm{S}$ & $\mathrm{R}$ & $\mathrm{R}$ & $\mathrm{R}$ & $\mathrm{R}$ & I & S & $\mathrm{S} * * *$ & S & $\mathrm{R}$ & $\mathrm{R}$ & $\mathrm{R}$ & $\mathrm{R}$ & $\mathrm{R}$ & $\mathrm{R}$ & $\mathrm{R}$ & $\mathrm{R}$ & $\mathrm{R}$ & $\mathrm{R}$ & I \\
\hline Natural penicillin & Penicillin-G & $\mathrm{R}$ & $\mathrm{R}$ & $\mathrm{R}$ & $\mathrm{R}$ & $\mathrm{R}$ & $\mathrm{R}$ & $\mathrm{R}$ & $\mathrm{R}$ & $\mathrm{R}$ & $\mathrm{R}$ & $\mathrm{R}$ & $\mathrm{R}$ & $\mathrm{R}$ & $\mathrm{R}$ & $\mathrm{R}$ & $\mathrm{R}$ & $\mathrm{R}$ & $\mathrm{R}$ & $\mathrm{R}$ & $\mathrm{R}$ \\
\hline Aminopenicillin & Ampicillin & $\mathrm{S}$ & I & $\mathrm{S}$ & $\mathrm{S}$ & $\mathrm{R}$ & $\mathrm{S}$ & $\mathrm{R}$ & $\mathrm{S}$ & $\mathrm{R}$ & $\mathrm{R}$ & $\mathrm{R}$ & $\mathrm{R}$ & $\mathrm{R}$ & $\mathrm{R}$ & $\mathrm{R}$ & $\mathrm{R}$ & $\mathrm{R}$ & $\mathrm{R}$ & I & $\mathrm{R}$ \\
\hline Carboxypenicillin & Ticarcillin & S & S & $\mathrm{S}$ & $\mathrm{R}$ & $\mathrm{R}$ & $\mathrm{S}$ & $\mathrm{R}$ & $\mathrm{S}$ & $\mathrm{S}$ & $\mathrm{R}$ & $\mathrm{R}$ & $\mathrm{R}$ & $\mathrm{R}$ & $\mathrm{R}$ & $\mathrm{R}$ & $\mathrm{R}$ & $\mathrm{R}$ & $\mathrm{R}$ & I & $\mathrm{R}$ \\
\hline Ureidopenicillin & Pipracillin & $S$ & I & $\mathrm{S}$ & $\mathrm{R}$ & $\mathrm{R}$ & I & $\mathrm{R}$ & $\mathrm{S}$ & S & $\mathrm{R}$ & $\mathrm{R}$ & $\mathrm{R}$ & $\mathrm{R}$ & $\mathrm{R}$ & $\mathrm{R}$ & $\mathrm{R}$ & $\mathrm{R}$ & $\mathrm{R}$ & $\mathrm{R}$ & $\mathrm{R}$ \\
\hline Polypeptide & Polymyxin-B & $\mathrm{R}$ & $\mathrm{R}$ & $\mathrm{R}$ & $\mathrm{R}$ & $\mathrm{S}$ & $\mathrm{R}$ & S & $\mathrm{S}$ & $\mathrm{R}$ & $\mathrm{S}$ & $\mathrm{S}$ & S & S & S & S & $\mathrm{R}$ & S & S & S & $\mathrm{R}$ \\
\hline Monobactums & Aztreonam & S & $\mathrm{R}$ & $\mathrm{S}$ & I & $\mathrm{R}$ & $\mathrm{S}$ & S & I & $\mathrm{R}$ & $\mathrm{R}$ & $\mathrm{R}$ & $\mathrm{R}$ & $\mathrm{R}$ & $\mathrm{R}$ & $\mathrm{R}$ & $\mathrm{R}$ & $\mathrm{R}$ & $\mathrm{R}$ & I & $\mathrm{R}$ \\
\hline Carbapenems & Meropenam & $\mathrm{S}$ & S & S & I & $\mathrm{R}$ & $\mathrm{S}$ & $\mathrm{R}$ & $\mathrm{S}$ & $\mathrm{R}$ & $\mathrm{R}$ & $\mathrm{R}$ & $\mathrm{R}$ & $\mathrm{R}$ & $\mathrm{R}$ & $\mathrm{R}$ & $\mathrm{R}$ & $\mathrm{R}$ & $\mathrm{R}$ & I & $\mathrm{S}$ \\
\hline Glycopeptides & Vancomycin & $\mathrm{R}$ & $\mathrm{R}$ & $\mathrm{R}$ & $\mathrm{R}$ & $\mathrm{R}$ & $\mathrm{R}$ & $\mathrm{R}$ & $\mathrm{R}$ & $\mathrm{R}$ & $\mathrm{R}$ & $\mathrm{R}$ & $\mathrm{R}$ & $\mathrm{R}$ & $\mathrm{R}$ & $\mathrm{R}$ & $\mathrm{R}$ & $\mathrm{R}$ & $\mathrm{R}$ & $\mathrm{R}$ & $\mathrm{R}$ \\
\hline Aminoglycoside & Kanamycin & $\mathrm{R}$ & $\mathrm{R}$ & I & $\mathrm{R}$ & $\mathrm{R}$ & $\mathrm{R}$ & $\mathrm{R}$ & $\mathrm{R}$ & $\mathrm{R}$ & $\mathrm{R}$ & $\mathrm{R}$ & $\mathrm{R}$ & $\mathrm{R}$ & $\mathrm{R}$ & $\mathrm{R}$ & $\mathrm{R}$ & $\mathrm{R}$ & $\mathrm{R}$ & I & $\mathrm{R}$ \\
\hline Aminoglycoside & Amikacin & $\mathrm{R}$ & $\mathrm{R}$ & $\mathrm{R}$ & $\mathrm{R}$ & $\mathrm{R}$ & $\mathrm{R}$ & $\mathrm{R}$ & $\mathrm{R}$ & $\mathrm{R}$ & $\mathrm{R}$ & $\mathrm{R}$ & $\mathrm{R}$ & $\mathrm{R}$ & $\mathrm{R}$ & $\mathrm{R}$ & $\mathrm{R}$ & $\mathrm{R}$ & $\mathrm{R}$ & I & $\mathrm{R}$ \\
\hline Aminoglycoside & Streptomycin & I & I & $\mathrm{S}$ & $\mathrm{R}$ & I & I & $\mathrm{R}$ & $\mathrm{S}$ & I & $\mathrm{S}$ & $\mathrm{S}$ & S & S & S & S & $\mathrm{S}$ & S & $\mathrm{S}$ & I & $\mathrm{R}$ \\
\hline Natural tetracycline & Tetracycline & S & S & $\mathrm{S}$ & $\mathrm{R}$ & $\mathrm{R}$ & I & $\mathrm{R}$ & $\mathrm{R}$ & S & $\mathrm{S}$ & S & S & S & S & S & I & S & S & $\mathrm{R}$ & $\mathrm{R}$ \\
\hline $\begin{array}{c}\begin{array}{c}\text { Semisynthetic } \\
\text { tetracycline }\end{array} \\
\end{array}$ & Doxycycline & I & S & $\mathrm{S}$ & $\mathrm{R}$ & $\mathrm{R}$ & $S$ & $\mathrm{R}$ & I & $S$ & $\mathrm{~S}$ & S & $S$ & $\mathrm{~S}$ & S & S & $S$ & S & $S$ & $\mathrm{R}$ & $\mathrm{S}$ \\
\hline Lincosamides & Clindamycin & $\mathrm{R}$ & $\mathrm{R}$ & $\mathrm{R}$ & $\mathrm{R}$ & $\mathrm{R}$ & $\mathrm{R}$ & $\mathrm{R}$ & $\mathrm{R}$ & $\mathrm{R}$ & $\mathrm{R}$ & $\mathrm{R}$ & $\mathrm{R}$ & $\mathrm{R}$ & $\mathrm{R}$ & $\mathrm{R}$ & $\mathrm{R}$ & $\mathrm{R}$ & $\mathrm{R}$ & $\mathrm{R}$ & $\mathrm{R}$ \\
\hline Macrolides(50-S) & Erythromycin & $\mathrm{R}$ & $\mathrm{R}$ & $\mathrm{R}$ & $\mathrm{R}$ & $\mathrm{R}$ & $\mathrm{R}$ & $\mathrm{R}$ & $\mathrm{R}$ & $\mathrm{R}$ & $\mathrm{R}$ & $\mathrm{R}$ & $\mathrm{R}$ & $\mathrm{R}$ & $\mathrm{R}$ & $\mathrm{R}$ & $\mathrm{R}$ & $\mathrm{R}$ & $\mathrm{R}$ & I & I \\
\hline Phenicoles(50-S) & $\begin{array}{c}\text { Chloramphenic } \\
\text { ol }\end{array}$ & $\mathrm{R}$ & S & $\mathrm{R}$ & $\mathrm{S}$ & $\mathrm{S}$ & $\mathrm{S}$ & S & $\mathrm{S}$ & $\mathrm{S}$ & $\mathrm{S}$ & $\mathrm{S}$ & S & S & S & S & $\mathrm{S}$ & S & $\mathrm{S}$ & $\mathrm{S}$ & $\mathrm{R}$ \\
\hline $1^{\text {st }}$ gen quinolone & Nalidixic acid & $S$ & I & $\mathrm{S}$ & I & $\mathrm{R}$ & $\mathrm{S}$ & I & $\mathrm{S}$ & $S$ & $\mathrm{R}$ & $\mathrm{R}$ & $\mathrm{R}$ & $\mathrm{R}$ & $\mathrm{R}$ & $\mathrm{R}$ & $\mathrm{R}$ & $\mathrm{R}$ & $\mathrm{R}$ & $\mathrm{R}$ & $\mathrm{S}$ \\
\hline $2^{\text {nd }}$ gen quinolone & Ciprofloxacin & $\mathrm{S}$ & S & $\mathrm{S}$ & S & $\mathrm{S}$ & $\mathrm{S}$ & $\mathrm{R}$ & $\mathrm{S}$ & S & $\mathrm{R}$ & $\mathrm{R}$ & $\mathrm{R}$ & $\mathrm{R}$ & $\mathrm{R}$ & $\mathrm{R}$ & $\mathrm{R}$ & $\mathrm{R}$ & $\mathrm{R}$ & I & $\mathrm{R}$ \\
\hline $3^{\text {rd }}$ gen quinolone & Levofloxacin & S & S & $\mathrm{S}$ & I & $\mathrm{S}$ & $\mathrm{S}$ & I & $\mathrm{S}$ & $\mathrm{R}$ & $\mathrm{R}$ & $\mathrm{R}$ & $\mathrm{R}$ & $\mathrm{R}$ & $\mathrm{R}$ & $\mathrm{R}$ & $\mathrm{R}$ & $\mathrm{R}$ & $\mathrm{R}$ & $S$ & I \\
\hline $\begin{array}{c}\text { RNA synthesis } \\
\text { inhibitor } \\
\end{array}$ & Rifampicin & $\mathrm{R}$ & $\mathrm{R}$ & $\mathrm{S}$ & $\mathrm{R}$ & $\mathrm{R}$ & $\mathrm{R}$ & $\mathrm{R}$ & $\mathrm{R}$ & $\mathrm{R}$ & $\mathrm{R}$ & $\mathrm{R}$ & $\mathrm{R}$ & $\mathrm{R}$ & $\mathrm{R}$ & $\mathrm{R}$ & $\mathrm{R}$ & $\mathrm{R}$ & $\mathrm{R}$ & $\mathrm{R}$ & $\mathrm{R}$ \\
\hline Sulphonamide & Trimethoprim & $\mathrm{S}$ & $\mathrm{S}$ & $\mathrm{S}$ & $\mathrm{R}$ & $\mathrm{R}$ & $\mathrm{S}$ & $\mathrm{R}$ & $\mathrm{S}$ & $\mathrm{S}$ & $\mathrm{R}$ & $\mathrm{R}$ & $\mathrm{R}$ & $\mathrm{R}$ & $\mathrm{R}$ & $\mathrm{R}$ & $\mathrm{R}$ & $\mathrm{R}$ & $\mathrm{R}$ & $\mathrm{R}$ & $\mathrm{S}$ \\
\hline $\begin{array}{c}\text { Combination of } \\
\text { sulpha }+ \text { trimetho. }\end{array}$ & Cotrimoxazole & S & S & $\mathrm{S}$ & $\mathrm{R}$ & $\mathrm{R}$ & $\mathrm{S}$ & $\mathrm{R}$ & $\mathrm{S}$ & $\mathrm{S}$ & $\mathrm{R}$ & $\mathrm{R}$ & $\mathrm{R}$ & $\mathrm{R}$ & $\mathrm{R}$ & $\mathrm{R}$ & $\mathrm{R}$ & $\mathrm{R}$ & $\mathrm{R}$ & $\mathrm{R}$ & $\mathrm{S}$ \\
\hline
\end{tabular}

$* \mathrm{R}=$ Resistant,$* * \mathrm{I}=$ Intermediate,$* * * \mathrm{~S}=$ Sensitive 
Table.4 Antibiogram of different isolates of Escherichia coli from pig and poultry

\begin{tabular}{|c|c|c|c|c|c|c|c|c|c|c|c|c|c|c|c|c|c|c|c|c|c|c|c|}
\hline Class of antibiotics & Antibiotics & $\begin{array}{l}\mathbf{P} \\
\mathbf{2}\end{array}$ & $\begin{array}{l}P \\
4\end{array}$ & $\begin{array}{l}\mathbf{P} \\
\mathbf{5}\end{array}$ & $\begin{array}{l}P \\
6\end{array}$ & $\begin{array}{l}\mathbf{P} \\
9\end{array}$ & $\begin{array}{l}\mathbf{P} \\
10\end{array}$ & $\begin{array}{l}\mathbf{P} \\
12\end{array}$ & $\begin{array}{l}\mathbf{P} \\
15\end{array}$ & $\begin{array}{l}\mathbf{P} \\
16\end{array}$ & $\begin{array}{l}\mathbf{P} \\
19\end{array}$ & $\begin{array}{c}\mathbf{P} \\
21\end{array}$ & $\begin{array}{c}\mathbf{P} \\
22\end{array}$ & $\begin{array}{l}\text { Pou } \\
\text { F2 }\end{array}$ & $\begin{array}{l}\text { Pou } \\
\text { F3 }\end{array}$ & $\begin{array}{l}\text { Pou } \\
\text { F4 }\end{array}$ & $\begin{array}{l}\text { Pou } \\
\text { S9 }\end{array}$ & $\begin{array}{c}\text { PouS } \\
10\end{array}$ & $\begin{array}{c}\text { PouS } \\
11\end{array}$ & $\begin{array}{c}\text { PouS } \\
12\end{array}$ & $\begin{array}{c}\text { PouS } \\
13\end{array}$ & $\begin{array}{c}\text { PouS } \\
14\end{array}$ & $\begin{array}{c}\text { PouS } \\
15\end{array}$ \\
\hline $1^{\text {st }}$ gen. cephalosporin & Cefazolin & $\mathrm{R}$ & $\mathrm{R}$ & $\mathrm{R}$ & $\mathrm{R}$ & $\mathrm{R}$ & $\mathrm{R}$ & $\mathrm{R}$ & $\mathrm{R}$ & $\mathrm{R}$ & $\mathrm{R}$ & $\mathrm{R}$ & $\mathrm{S}$ & $\mathrm{R}$ & $\mathrm{I} * *$ & $\mathrm{R}$ & $\mathrm{R}$ & $\mathrm{R}$ & $\mathrm{R}$ & $\mathrm{R}$ & $\mathrm{R}$ & $\mathrm{R}$ & $\mathrm{R}$ \\
\hline $2^{\text {nd }}$ gen. cephalosporin & Cefuroxime & $\mathrm{R}$ & $\mathrm{R}$ & $\mathrm{R}$ & $\mathrm{R}$ & $\mathrm{R}$ & $\mathrm{R}$ & $\mathrm{R}$ & $\mathrm{R}$ & $\mathrm{R}$ & $\mathrm{R}$ & $\mathrm{R}$ & $\mathrm{R}$ & $\mathrm{R}$ & $\mathrm{R} *$ & $\mathrm{R}$ & $\mathrm{R}$ & $\mathrm{R}$ & $\mathrm{R}$ & $\mathrm{R}$ & $\mathrm{R}$ & $\mathrm{R}$ & $\mathrm{R}$ \\
\hline $\begin{array}{c}3^{\text {rd }} \text { gen. } \\
\text { cephalosporin } \\
\end{array}$ & Ceftazidime & I & $\mathrm{R}$ & $\mathrm{R}$ & $\mathrm{R}$ & $\mathrm{R}$ & $\mathrm{S}$ & $\mathrm{R}$ & $\mathrm{R}$ & I & $\mathrm{S}$ & $\mathrm{R}$ & $\mathrm{S}$ & $\mathrm{R}$ & $\mathrm{S} * * *$ & $\mathrm{R}$ & S & $\mathrm{R}$ & $\mathrm{R}$ & S & S & $\mathrm{R}$ & $\mathrm{R}$ \\
\hline $3^{\text {rd }}$ gen. cephalosporin & Cefotaxime & $\mathrm{R}$ & $\mathrm{R}$ & I & $\mathrm{R}$ & I & I & $\mathrm{R}$ & I & $\mathrm{S}$ & $\mathrm{R}$ & $\mathrm{R}$ & $\mathrm{R}$ & $\mathrm{R}$ & $\mathrm{S}$ & I & S & $\mathrm{R}$ & $\mathrm{R}$ & S & $\mathrm{R}$ & S & I \\
\hline $4^{\text {th }}$ gen. cephalosporin & Cefipime & $\mathrm{S}$ & $\mathrm{S}$ & $\mathrm{R}$ & $\mathrm{S}$ & $S$ & $\mathrm{~S}$ & $\mathrm{~S}$ & $\mathrm{~S}$ & $\mathrm{~S}$ & $\mathrm{R}$ & I & $\mathrm{S}$ & $\mathrm{R}$ & S & S & S & $\mathrm{R}$ & I & I & $\mathrm{R}$ & $\mathrm{R}$ & $\mathrm{R}$ \\
\hline Natural penicillin & Penicillin-G & $\mathrm{R}$ & $\mathrm{R}$ & $\mathrm{R}$ & $\mathrm{R}$ & $\mathrm{R}$ & $\mathrm{R}$ & $\mathrm{R}$ & $\mathrm{R}$ & $\mathrm{R}$ & $\mathrm{R}$ & $\mathrm{R}$ & $\mathrm{R}$ & $\mathrm{R}$ & $\mathrm{R}$ & $\mathrm{R}$ & $\mathrm{R}$ & $\mathrm{R}$ & $\mathrm{R}$ & $\mathrm{R}$ & $\mathrm{R}$ & $\mathrm{R}$ & $\mathrm{R}$ \\
\hline Penicillinase stable & Oxacillin & $\mathrm{R}$ & $\mathrm{R}$ & $\mathrm{R}$ & $\mathrm{R}$ & $\mathrm{R}$ & $\mathrm{R}$ & $\mathrm{R}$ & $\mathrm{R}$ & $\mathrm{R}$ & $\mathrm{R}$ & $\mathrm{R}$ & $\mathrm{R}$ & $\mathrm{R}$ & $\mathrm{R}$ & $\mathrm{R}$ & $\mathrm{R}$ & $\mathrm{R}$ & $\mathrm{R}$ & $\mathrm{R}$ & $\mathrm{R}$ & $\mathrm{R}$ & $\mathrm{R}$ \\
\hline Aminopenicillin & Ampicillin & $\mathrm{S}$ & I & S & $\mathrm{R}$ & $\mathrm{R}$ & $\mathrm{R}$ & I & I & $\mathrm{R}$ & $\mathrm{S}$ & $\mathrm{S}$ & $\mathrm{S}$ & $\mathrm{R}$ & $S$ & $S$ & S & $\mathrm{R}$ & $\mathrm{R}$ & S & $\mathrm{R}$ & $\mathrm{R}$ & $\mathrm{R}$ \\
\hline Carboxypenicillin & Ticarcillin & $\mathrm{S}$ & I & I & I & $S$ & $\mathrm{~S}$ & $\mathrm{~S}$ & $\mathrm{~S}$ & $\mathrm{~S}$ & I & $\mathrm{S}$ & $\mathrm{S}$ & $\mathrm{R}$ & S & S & S & $\mathrm{R}$ & $\mathrm{R}$ & S & $S$ & S & $\mathrm{R}$ \\
\hline Ureidopenicillin & Pipracillin & $\mathrm{S}$ & I & I & $\mathrm{R}$ & I & $\mathrm{S}$ & $\mathrm{S}$ & I & $\mathrm{S}$ & I & I & I & $\mathrm{R}$ & S & $S$ & I & $\mathrm{R}$ & $\mathrm{R}$ & I & $\mathrm{R}$ & $\mathrm{R}$ & $\mathrm{R}$ \\
\hline Polypeptide & Polymyxin-B & $\mathrm{R}$ & $\mathrm{S}$ & S & $\mathrm{R}$ & $\mathrm{R}$ & $S$ & $\mathrm{~S}$ & $\mathrm{~S}$ & $\mathrm{~S}$ & $\mathrm{R}$ & $\mathrm{R}$ & $\mathrm{R}$ & $\mathrm{R}$ & S & S & $\mathrm{R}$ & S & $\mathrm{R}$ & $\mathrm{R}$ & $\mathrm{R}$ & $\mathrm{R}$ & $\mathrm{R}$ \\
\hline Monobactums & Aztreonam & I & $\mathrm{S}$ & S & $\mathrm{S}$ & I & $\mathrm{S}$ & I & I & $\mathrm{S}$ & $\mathrm{R}$ & I & I & $\mathrm{R}$ & S & S & S & $\mathrm{R}$ & I & S & S & I & $S$ \\
\hline Carbapenems & Meropenam & $\mathrm{S}$ & I & S & $\mathrm{R}$ & I & $\mathrm{S}$ & $\mathrm{R}$ & $\mathrm{S}$ & I & I & I & $\mathrm{R}$ & $\mathrm{S}$ & $\mathrm{R}$ & $\mathrm{R}$ & $\mathrm{R}$ & I & $\mathrm{R}$ & S & $\mathrm{R}$ & $\mathrm{R}$ & S \\
\hline Glycopeptides & Vancomycin & $\mathrm{R}$ & $\mathrm{R}$ & $\mathrm{R}$ & $\mathrm{R}$ & $\mathrm{R}$ & $\mathrm{R}$ & $\mathrm{R}$ & $\mathrm{R}$ & $\mathrm{R}$ & $\mathrm{R}$ & $\mathrm{R}$ & $\mathrm{R}$ & $\mathrm{R}$ & $\mathrm{R}$ & $\mathrm{R}$ & $\mathrm{R}$ & $\mathrm{R}$ & $\mathrm{R}$ & $\mathrm{R}$ & $\mathrm{R}$ & $\mathrm{R}$ & $\mathrm{R}$ \\
\hline Aminoglycoside & Kanamycin & I & I & $\mathrm{R}$ & $\mathrm{R}$ & I & I & I & $\mathrm{R}$ & I & $\mathrm{R}$ & $\mathrm{R}$ & I & I & $\mathrm{R}$ & $\mathrm{R}$ & I & $\mathrm{R}$ & $\mathrm{R}$ & $\mathrm{R}$ & $\mathrm{R}$ & $\mathrm{R}$ & $\mathrm{R}$ \\
\hline Aminoglycoside & Gentamicin & $\mathrm{R}$ & $\mathrm{S}$ & $\mathrm{R}$ & $\mathrm{R}$ & $S$ & $\mathrm{~S}$ & $\mathrm{~S}$ & $\mathrm{~S}$ & $\mathrm{~S}$ & $\mathrm{~S}$ & $\mathrm{~S}$ & $\mathrm{~S}$ & $S$ & $\mathrm{~S}$ & $S$ & S & I & $S$ & S & $S$ & $S$ & $S$ \\
\hline Aminoglycoside & Amikacin & I & $\mathrm{S}$ & I & $\mathrm{R}$ & I & $\mathrm{R}$ & $\mathrm{R}$ & I & $\mathrm{R}$ & I & $\mathrm{R}$ & $\mathrm{S}$ & $\mathrm{S}$ & $\mathrm{S}$ & $\mathrm{R}$ & $\mathrm{S}$ & S & $\mathrm{R}$ & $\mathrm{S}$ & $\mathrm{R}$ & $\mathrm{R}$ & S \\
\hline Aminoglycoside & Streptomycin & I & I & I & I & I & $\mathrm{S}$ & I & I & I & $\mathrm{S}$ & $\mathrm{R}$ & $\mathrm{S}$ & I & $\mathrm{R}$ & $\mathrm{R}$ & S & I & $\mathrm{R}$ & S & $\mathrm{R}$ & S & I \\
\hline Natural tetracycline & Tetracycline & $\mathrm{S}$ & $\mathrm{S}$ & S & $\mathrm{S}$ & $S$ & $\mathrm{~S}$ & $\mathrm{R}$ & $\mathrm{S}$ & $\mathrm{S}$ & $\mathrm{S}$ & $\mathrm{S}$ & $\mathrm{S}$ & $\mathrm{R}$ & $\mathrm{R}$ & $\mathrm{R}$ & I & $\mathrm{R}$ & $\mathrm{R}$ & $\mathrm{R}$ & $\mathrm{R}$ & $\mathrm{R}$ & $\mathrm{R}$ \\
\hline $\begin{array}{l}\text { Semisynthetic } \\
\text { tetracycline }\end{array}$ & Doxycycline & $\mathrm{S}$ & $\mathrm{S}$ & $\mathrm{S}$ & $\mathrm{S}$ & S & $\mathrm{S}$ & $\mathrm{S}$ & $\mathrm{S}$ & $\mathrm{S}$ & $\mathrm{S}$ & $\mathrm{S}$ & $\mathrm{S}$ & $\mathrm{R}$ & $\mathrm{R}$ & $\mathrm{R}$ & I & $\mathrm{R}$ & $\mathrm{R}$ & $\mathrm{R}$ & $\mathrm{R}$ & I & $\mathrm{R}$ \\
\hline Lincosamides & Clindamycin & $\mathrm{R}$ & $\mathrm{R}$ & $\mathrm{R}$ & $\mathrm{R}$ & $\mathrm{R}$ & $\mathrm{R}$ & $\mathrm{R}$ & $\mathrm{R}$ & $\mathrm{R}$ & $\mathrm{R}$ & $\mathrm{R}$ & $\mathrm{R}$ & $\mathrm{R}$ & $\mathrm{R}$ & $\mathrm{R}$ & $\mathrm{R}$ & $\mathrm{R}$ & $\mathrm{R}$ & $\mathrm{R}$ & $\mathrm{R}$ & $\mathrm{R}$ & $\mathrm{R}$ \\
\hline Macrolides(50-S) & Erythromycin & $\mathrm{R}$ & $\mathrm{R}$ & $\mathrm{R}$ & $\mathrm{R}$ & $\mathrm{R}$ & $\mathrm{R}$ & $\mathrm{R}$ & $\mathrm{R}$ & $\mathrm{R}$ & $\mathrm{R}$ & $\mathrm{R}$ & $\mathrm{R}$ & $\mathrm{R}$ & $\mathrm{R}$ & $\mathrm{R}$ & $\mathrm{R}$ & $\mathrm{R}$ & $\mathrm{R}$ & $\mathrm{R}$ & $\mathrm{R}$ & $\mathrm{R}$ & $\mathrm{R}$ \\
\hline Phenicoles(50-S) & Chlorampheniol & $\mathrm{S}$ & $\mathrm{S}$ & $\mathrm{S}$ & $\mathrm{S}$ & S & $\mathrm{S}$ & $\mathrm{S}$ & $\mathrm{S}$ & $\mathrm{S}$ & I & I & $\mathrm{S}$ & I & $\mathrm{S}$ & $\mathrm{S}$ & $\mathrm{S}$ & $\mathrm{R}$ & $\mathrm{R}$ & $\mathrm{S}$ & I & $\mathrm{S}$ & $\mathrm{S}$ \\
\hline $1^{\text {st }}$ gen quinolone & Nalidixic acid & I & I & $\mathrm{R}$ & $\mathrm{R}$ & $\mathrm{R}$ & $\mathrm{R}$ & $\mathrm{S}$ & I & I & $\mathrm{R}$ & $\mathrm{R}$ & $\mathrm{R}$ & $\mathrm{R}$ & $\mathrm{R}$ & $\mathrm{R}$ & $S$ & $\mathrm{R}$ & $\mathrm{R}$ & $S$ & $\mathrm{R}$ & $\mathrm{R}$ & $\mathrm{R}$ \\
\hline $2^{\text {nd }}$ gen quinolone & Ciprofloxacin & $\mathrm{S}$ & $\mathrm{S}$ & S & $\mathrm{S}$ & S & $\mathrm{S}$ & I & $\mathrm{S}$ & I & $\mathrm{S}$ & I & $\mathrm{S}$ & I & I & $\mathrm{R}$ & S & I & $\mathrm{R}$ & S & I & S & $\mathrm{R}$ \\
\hline $3^{\text {rd }}$ gen quinolone & Levofloxacin & $\mathrm{S}$ & $\mathrm{S}$ & S & $\mathrm{S}$ & S & $\mathrm{S}$ & $\mathrm{S}$ & $\mathrm{S}$ & $\mathrm{S}$ & $\mathrm{S}$ & $\mathrm{S}$ & I & $\mathrm{S}$ & $\mathrm{S}$ & $\mathrm{R}$ & S & S & $\mathrm{S}$ & S & S & I & $\mathrm{S}$ \\
\hline $\begin{array}{l}\text { RNA synthesis } \\
\text { inhibitor }\end{array}$ & Rifampicin & $\mathrm{R}$ & $\mathrm{R}$ & $\mathrm{R}$ & $\mathrm{R}$ & $\mathrm{R}$ & $\mathrm{R}$ & $\mathrm{R}$ & $\mathrm{R}$ & $\mathrm{R}$ & $\mathrm{R}$ & $\mathrm{R}$ & $\mathrm{R}$ & $\mathrm{R}$ & $\mathrm{R}$ & $\mathrm{R}$ & $\mathrm{R}$ & $\mathrm{R}$ & $\mathrm{R}$ & $\mathrm{R}$ & $\mathrm{R}$ & $\mathrm{R}$ & $\mathrm{R}$ \\
\hline Sulphonamide & Trimethoprim & $\mathrm{S}$ & $\mathrm{S}$ & S & $\mathrm{S}$ & S & $\mathrm{S}$ & I & $\mathrm{S}$ & $\mathrm{S}$ & $\mathrm{S}$ & $\mathrm{S}$ & $\mathrm{S}$ & $\mathrm{R}$ & $\mathrm{R}$ & S & S & $\mathrm{R}$ & $\mathrm{R}$ & S & $\mathrm{R}$ & S & $\mathrm{S}$ \\
\hline $\begin{array}{l}\text { Combination of } \\
\text { sulpha + trimetho. }\end{array}$ & Cotrimoxazole & $\mathrm{S}$ & $\mathrm{S}$ & $\mathrm{S}$ & $\mathrm{S}$ & S & $\mathrm{S}$ & $\mathrm{S}$ & $\mathrm{S}$ & $\mathrm{S}$ & $\mathrm{S}$ & $\mathrm{S}$ & $\mathrm{S}$ & $\mathrm{R}$ & $\mathrm{R}$ & S & S & $\mathrm{R}$ & $\mathrm{R}$ & S & S & $\mathrm{R}$ & $\mathrm{R}$ \\
\hline
\end{tabular}

$* \mathrm{R}=$ Resistant, $* * \mathrm{I}=$ Intermediate, $* * * \mathrm{~S}=$ Sensitive 
Table.5 Intensity of florescence of different Escherichia coli isolates at different concentrations of Ethidium Bromide

\begin{tabular}{|c|c|c|}
\hline Intensity & Isolates not showing fluorescence & Isolates showing florescence \\
\hline \multicolumn{3}{|c|}{ Result of florescence at $0.5 \mathrm{mg} / \mathrm{l}$ concentration } \\
\hline Human & $\begin{array}{l}\text { HU2, HU4, HU7, HU12, HU13, HU15, HU17, HU18, } \\
\text { HU20, HU22, HU23, HU24, HU27, HU29, HU30, E.H. }\end{array}$ & HU3, HS10,HU14, E.J. \\
\hline Pig & $\mathrm{P} 2, \mathrm{P} 4, \mathrm{P} 10, \mathrm{P} 12, \mathrm{P} 15, \mathrm{P} 19, \mathrm{P} 21, \mathrm{P} 22$ & P5, P6, P9, P16 \\
\hline Poultry & $\begin{array}{l}\text { PouF2, PouF3, PouF4, PouS9, PouS10, PouS11, PouS12, } \\
\text { PouS13, PouS14, PouS15 }\end{array}$ & NIL \\
\hline \multicolumn{3}{|c|}{ Result of florescence at $1 \mathrm{mg} / \mathrm{l}$ concentration } \\
\hline Human & $\begin{array}{l}\text { HU2, HU7, HU17, HU18, HU20, HU22, HU23, HU24, } \\
\text { HU27, HU29, HU30, E.H. }\end{array}$ & HU3, HU4, HS10, HU12, HU13, HU14, HU15, E.J. \\
\hline Pig & $\mathrm{P} 2, \mathrm{P} 4, \mathrm{P} 10, \mathrm{P} 12, \mathrm{P} 15, \mathrm{P} 21$ & P5, P6, P9, P16, P19, P22 \\
\hline Poultry & PouF2, PouF3, PouF4, PouS9, PouS10, PouS12, PouS14 & PouS11, PouS13, PouS15 \\
\hline \multicolumn{3}{|c|}{ Result of florescence at $1.5 \mathrm{mg} / \mathrm{l}$ concentration } \\
\hline Human & HU17, HU18, HU22, HU23, HU24, HU27, HU29, HU30 & $\begin{array}{l}\text { HU2, HU3, HU4, HU7, HS10, HU12, HU13, HU14, HU15, } \\
\text { HU20, E.J., E.H. }\end{array}$ \\
\hline Pig & $\mathrm{P} 2, \mathrm{P} 4, \mathrm{P} 12, \mathrm{P} 15$ & P5, P6, P9, P10, P16, P19, P21, P22 \\
\hline Poultry & PouS9, PouS12 & $\begin{array}{l}\text { PouF2, PouF3, PouF4, PouS10, PouS11, PouS13, PouS14, } \\
\text { PouS15 }\end{array}$ \\
\hline \multicolumn{3}{|c|}{ Result of florescence at $2 \mathrm{mg} / \mathrm{l}$ concentration } \\
\hline Human & HU17, HU18, HU22, HU23, HU24, HU27, HU29, HU30 & $\begin{array}{l}\text { HU2, HU3, HU4, HU7, HS10, HU12, HU13, HU14, HU15, } \\
\text { HU20, E.J., E.H. }\end{array}$ \\
\hline Pig & $\mathrm{P} 12, \mathrm{P} 15$ & P2, P4, P5, P6, P9, P10, P16, P19, P21, P22 \\
\hline Poultry & PouS9, PouS12 & $\begin{array}{l}\text { PouF2, PouF3, PouF4, PouS10, PouS11, PouS13, PouS14, } \\
\text { PouS15 }\end{array}$ \\
\hline \multicolumn{3}{|c|}{ Result of florescence at $2.5 \mathrm{mg} / \mathrm{l}$ concentration } \\
\hline Human & HU17, HU18, HU22, HU23, HU24, HU27, HU29, HU30 & $\begin{array}{l}\text { HU2, HU3, HU4, HU7, HS10, HU12, HU13, HU14, HU15, } \\
\text { HU20, E.J., E.H. }\end{array}$ \\
\hline Pig & NIL & $\mathrm{P} 2, \mathrm{P} 4, \mathrm{P} 5, \mathrm{P} 6, \mathrm{P} 9, \mathrm{P} 10, \mathrm{P} 12, \mathrm{P} 15, \mathrm{P} 16, \mathrm{P} 19, \mathrm{P} 21, \mathrm{P} 22$ \\
\hline Poultry & PouS9, PouS12 & $\begin{array}{l}\text { PouF2, PouF3, PouF4, PouS10, PouS11, PouS13, PouS14, } \\
\text { PouS15 }\end{array}$ \\
\hline
\end{tabular}


Table.6 Antibiogram of different isolates of Escherichia coli on Sub-culturing

\begin{tabular}{|c|c|c|c|c|c|c|c|c|c|c|c|c|c|c|c|c|c|c|c|c|c|c|c|c|c|c|c|c|}
\hline \multirow{2}{*}{$\begin{array}{l}\text { Subc } \\
\text { ulture }\end{array}$} & \multicolumn{7}{|c|}{ HU-2 } & \multicolumn{7}{|c|}{ HU-3 } & \multicolumn{7}{|c|}{ HU-4 } & \multicolumn{7}{|c|}{ HU-7 } \\
\hline & $\begin{array}{l}\mathbf{T} \\
\mathbf{E}\end{array}$ & $\begin{array}{l}\mathrm{C} \\
\mathbf{O}\end{array}$ & $\begin{array}{c}\mathbf{A} \\
\mathbf{M} \\
\mathbf{P}\end{array}$ & C & $\begin{array}{l}\mathbf{M} \\
\mathbf{R} \\
\mathbf{P}\end{array}$ & $\begin{array}{c}\mathbf{C} \\
\mathbf{P} \\
\mathbf{M}\end{array}$ & $\begin{array}{l}\mathbf{L} \\
\mathbf{E}\end{array}$ & $\begin{array}{l}\mathbf{T} \\
\mathbf{E}\end{array}$ & $\begin{array}{l}\mathbf{C} \\
\mathbf{O}\end{array}$ & $\begin{array}{c}\mathbf{A} \\
\mathbf{M} \\
\mathbf{P}\end{array}$ & C & $\begin{array}{l}\mathbf{M} \\
\mathbf{R} \\
\mathbf{P}\end{array}$ & $\begin{array}{c}\mathbf{C} \\
\mathbf{P} \\
\mathbf{M}\end{array}$ & $\begin{array}{l}\mathbf{L} \\
\mathbf{E}\end{array}$ & $\begin{array}{l}\mathbf{T} \\
\mathbf{E}\end{array}$ & $\begin{array}{l}\text { C } \\
\mathbf{O}\end{array}$ & $\begin{array}{c}\mathbf{A} \\
\mathbf{M} \\
\mathbf{P}\end{array}$ & C & $\begin{array}{c}\mathbf{M} \\
\mathbf{R} \\
\mathbf{P}\end{array}$ & $\begin{array}{c}\mathbf{C} \\
\mathbf{P} \\
\mathbf{M}\end{array}$ & $\begin{array}{l}\mathbf{L} \\
\mathbf{E}\end{array}$ & $\begin{array}{l}\mathbf{T} \\
\mathbf{E}\end{array}$ & $\begin{array}{l}\text { C } \\
\mathbf{O}\end{array}$ & $\begin{array}{c}\mathbf{A} \\
\mathbf{M} \\
\mathbf{P}\end{array}$ & C & $\begin{array}{l}\mathbf{M} \\
\mathbf{R} \\
\mathbf{P}\end{array}$ & $\begin{array}{c}\mathbf{C} \\
\mathbf{P} \\
\mathbf{M}\end{array}$ & $\begin{array}{l}\mathbf{L} \\
\mathbf{E}\end{array}$ \\
\hline Sub-1 & I & $S$ & I & I & $\mathrm{R}$ & I & $\mathrm{S}$ & $S$ & $\mathrm{~S}$ & $\mathrm{R}$ & $\mathrm{S}$ & $S$ & $\mathrm{R}$ & $\mathrm{S}$ & $S$ & $\mathrm{R}$ & $S$ & $\mathrm{R}$ & I & $\mathrm{R}$ & I & $\mathrm{R}$ & $\mathrm{R}$ & $S$ & $\mathrm{~S}$ & I & $\mathrm{R}$ & I \\
\hline Sub-2 & I & $S$ & I & $\mathrm{S}$ & $\mathrm{R}$ & S & S & $S$ & $\mathrm{~S}$ & I & $\mathrm{S}$ & S & $\mathrm{R}$ & $\mathrm{S}$ & $S$ & $\mathrm{~S}$ & S & $\mathrm{S}$ & I & $\mathrm{R}$ & S & $\mathrm{R}$ & $\mathrm{R}$ & S & $\mathrm{S}$ & S & $\mathrm{R}$ & $\mathrm{S}$ \\
\hline Sub-3 & I & $S$ & I & $\mathrm{S}$ & I & $\mathrm{S}$ & $\mathrm{S}$ & $S$ & $\mathrm{~S}$ & $\mathrm{~S}$ & $\mathrm{~S}$ & S & $\mathrm{R}$ & $\mathrm{S}$ & $S$ & $\mathrm{~S}$ & $S$ & $\mathrm{~S}$ & I & $\mathrm{R}$ & $\mathrm{S}$ & $\mathrm{R}$ & $\mathrm{R}$ & $\mathrm{R}$ & $\mathrm{S}$ & $\mathrm{S}$ & $\mathrm{R}$ & $\mathrm{S}$ \\
\hline Sub-4 & I & S & S & $\mathrm{S}$ & I & S & S & S & $\mathrm{S}$ & $\mathrm{S}$ & $\mathrm{S}$ & S & $\mathrm{R}$ & $\mathrm{S}$ & S & S & S & $\mathrm{S}$ & I & $\mathrm{R}$ & S & $\mathrm{R}$ & $\mathrm{R}$ & $\mathrm{R}$ & $\mathrm{S}$ & S & $\mathrm{R}$ & $\mathrm{S}$ \\
\hline Sub-5 & I & $S$ & $\mathrm{~S}$ & $\mathrm{~S}$ & $S$ & $\mathrm{~S}$ & $\mathrm{~S}$ & $\mathrm{~S}$ & $\mathrm{~S}$ & $\mathrm{~S}$ & $\mathrm{~S}$ & $\mathrm{~S}$ & $\mathrm{R}$ & $\mathrm{S}$ & $S$ & $\mathrm{~S}$ & $S$ & $\mathrm{~S}$ & I & $\mathrm{R}$ & S & $\mathrm{R}$ & $\mathrm{R}$ & $\mathrm{R}$ & $\mathrm{S}$ & S & $\mathrm{R}$ & $\mathrm{S}$ \\
\hline \multirow{2}{*}{$\begin{array}{l}\text { Subc } \\
\text { ulture }\end{array}$} & \multicolumn{7}{|c|}{ HS-10 } & \multicolumn{7}{|c|}{ HU-12 } & \multicolumn{7}{|c|}{ HU-13 } & \multicolumn{7}{|c|}{ HU-14 } \\
\hline & $\begin{array}{l}\mathbf{T} \\
\mathbf{E}\end{array}$ & $\begin{array}{l}\text { C } \\
\mathbf{O}\end{array}$ & $\begin{array}{c}\mathbf{A} \\
\mathbf{M} \\
\mathbf{P}\end{array}$ & C & $\begin{array}{l}\mathbf{M} \\
\mathbf{R} \\
\mathbf{P}\end{array}$ & $\begin{array}{c}\mathbf{C} \\
\mathbf{P} \\
\mathbf{M}\end{array}$ & $\begin{array}{l}\mathbf{L} \\
\mathbf{E}\end{array}$ & $\begin{array}{l}\mathbf{T} \\
\mathbf{E}\end{array}$ & $\begin{array}{l}\text { C } \\
\text { O }\end{array}$ & $\begin{array}{c}\mathbf{A} \\
\mathbf{M} \\
\mathbf{P}\end{array}$ & C & $\begin{array}{l}\mathbf{M} \\
\mathbf{R} \\
\mathbf{P}\end{array}$ & $\begin{array}{c}\mathbf{C} \\
\mathbf{P} \\
\mathbf{M}\end{array}$ & $\begin{array}{l}\mathbf{L} \\
\mathbf{E}\end{array}$ & $\begin{array}{l}\mathbf{T} \\
\mathbf{E}\end{array}$ & $\begin{array}{l}\text { C } \\
\text { O }\end{array}$ & $\begin{array}{c}\mathbf{A} \\
\mathbf{M} \\
\mathbf{P}\end{array}$ & C & $\begin{array}{c}\mathbf{M} \\
\mathbf{R} \\
\mathbf{P}\end{array}$ & $\begin{array}{c}\mathbf{C} \\
\mathbf{P} \\
\mathbf{M}\end{array}$ & $\begin{array}{l}\mathbf{L} \\
\mathbf{E}\end{array}$ & $\begin{array}{l}\mathbf{T} \\
\mathbf{E}\end{array}$ & $\begin{array}{l}\text { C } \\
\text { O }\end{array}$ & $\begin{array}{c}\mathbf{A} \\
\mathbf{M} \\
\mathbf{P}\end{array}$ & C & $\begin{array}{l}\mathbf{M} \\
\mathbf{R} \\
\mathbf{P}\end{array}$ & $\begin{array}{c}\mathbf{C} \\
\mathbf{P} \\
\mathbf{M}\end{array}$ & $\begin{array}{l}\mathbf{L} \\
\mathbf{E}\end{array}$ \\
\hline Sub-1 & $\mathrm{R}$ & $\mathrm{R}$ & $\mathrm{R}$ & $\mathrm{S}$ & $\mathrm{R}$ & $\mathrm{R}$ & $\mathrm{S}$ & $\mathrm{R}$ & $\mathrm{S}$ & $\mathrm{R}$ & I & $\mathrm{R}$ & $S$ & 1 & $\mathrm{R}$ & $\mathrm{R}$ & $\mathrm{R}$ & $S$ & $\mathrm{R}$ & $\mathrm{S}$ & I & $\mathrm{R}$ & $\mathrm{S}$ & $S$ & $\mathrm{~S}$ & I & $\mathrm{R}$ & $\mathrm{S}$ \\
\hline Sub-2 & $\mathrm{R}$ & $\mathrm{R}$ & $\mathrm{R}$ & $\mathrm{S}$ & $\mathrm{R}$ & $\mathrm{R}$ & S & S & $\mathrm{S}$ & I & $\mathrm{S}$ & I & $\mathrm{S}$ & $\mathrm{S}$ & $\mathrm{R}$ & $\mathrm{R}$ & $\mathrm{R}$ & $\mathrm{S}$ & $\mathrm{R}$ & $\mathrm{S}$ & I & $\mathrm{R}$ & S & $S$ & $\mathrm{~S}$ & S & $\mathrm{R}$ & $\mathrm{S}$ \\
\hline Sub-3 & $\mathrm{R}$ & $\mathrm{R}$ & $\mathrm{R}$ & $\mathrm{S}$ & $S$ & $\mathrm{R}$ & $\mathrm{S}$ & $S$ & $\mathrm{~S}$ & I & $\mathrm{S}$ & $S$ & $S$ & $\mathrm{~S}$ & $\mathrm{R}$ & $\mathrm{R}$ & $\mathrm{R}$ & $S$ & $\mathrm{R}$ & $S$ & I & $\mathrm{R}$ & $\mathrm{S}$ & $S$ & $\mathrm{~S}$ & $S$ & $\mathrm{R}$ & $\mathrm{S}$ \\
\hline Sub-4 & $\mathrm{R}$ & $\mathrm{R}$ & $\mathrm{R}$ & $\mathrm{S}$ & S & $\mathrm{R}$ & $\mathrm{S}$ & $\mathrm{S}$ & $\mathrm{S}$ & I & $\mathrm{S}$ & S & $\mathrm{S}$ & $\mathrm{S}$ & $\mathrm{R}$ & $\mathrm{R}$ & $\mathrm{R}$ & $\mathrm{S}$ & $\mathrm{R}$ & S & I & $\mathrm{R}$ & $\mathrm{S}$ & S & $\mathrm{S}$ & S & $\mathrm{R}$ & $\mathrm{S}$ \\
\hline Sub-5 & $\mathrm{R}$ & $\mathrm{R}$ & $\mathrm{R}$ & $\mathrm{S}$ & S & $\mathrm{R}$ & S & S & $\mathrm{S}$ & I & $\mathrm{S}$ & S & S & S & $\mathrm{R}$ & $\mathrm{R}$ & $\mathrm{R}$ & $S$ & I & S & S & $\mathrm{R}$ & S & S & $\mathrm{S}$ & S & $\mathrm{R}$ & $\mathrm{S}$ \\
\hline \multirow{2}{*}{$\begin{array}{l}\text { Subc } \\
\text { ulture }\end{array}$} & \multicolumn{7}{|c|}{ HU-15 } & \multicolumn{7}{|c|}{ HU-17 } & \multicolumn{7}{|c|}{ HU-18 } & \multicolumn{7}{|c|}{ HU-20 } \\
\hline & $\begin{array}{l}\mathbf{T} \\
\mathbf{E}\end{array}$ & $\begin{array}{l}\text { C } \\
\mathbf{O}\end{array}$ & $\begin{array}{c}\mathbf{A} \\
\mathbf{M} \\
\mathbf{P}\end{array}$ & C & $\begin{array}{l}\mathbf{M} \\
\mathbf{R} \\
\mathbf{P}\end{array}$ & $\begin{array}{c}\mathbf{C} \\
\mathbf{P} \\
\mathbf{M}\end{array}$ & $\begin{array}{l}\mathbf{L} \\
\mathbf{E}\end{array}$ & $\begin{array}{l}\mathbf{T} \\
\mathbf{E}\end{array}$ & $\begin{array}{l}\text { C } \\
\mathbf{O}\end{array}$ & $\begin{array}{c}\mathbf{A} \\
\mathbf{M} \\
\mathbf{P}\end{array}$ & C & $\begin{array}{l}\mathbf{M} \\
\mathbf{R} \\
\mathbf{P}\end{array}$ & $\begin{array}{c}\mathbf{C} \\
\mathbf{P} \\
\mathbf{M}\end{array}$ & $\begin{array}{l}\mathbf{L} \\
\mathbf{E}\end{array}$ & $\begin{array}{l}\mathbf{T} \\
\mathbf{E}\end{array}$ & $\begin{array}{l}\text { C } \\
\text { O }\end{array}$ & $\begin{array}{c}\mathbf{A} \\
\mathbf{M} \\
\mathbf{P}\end{array}$ & C & $\begin{array}{c}\mathbf{M} \\
\mathbf{R} \\
\mathbf{P}\end{array}$ & $\begin{array}{c}\mathbf{C} \\
\mathbf{P} \\
\mathbf{M}\end{array}$ & $\begin{array}{l}\mathbf{L} \\
\mathbf{E}\end{array}$ & $\begin{array}{l}\mathbf{T} \\
\mathbf{E}\end{array}$ & $\begin{array}{l}\text { C } \\
\mathbf{O}\end{array}$ & $\begin{array}{c}\mathbf{A} \\
\mathbf{M} \\
\mathbf{P}\end{array}$ & C & $\begin{array}{l}\mathbf{M} \\
\mathbf{R} \\
\mathbf{P}\end{array}$ & $\begin{array}{c}\mathbf{C} \\
\mathbf{P} \\
\mathbf{M}\end{array}$ & $\begin{array}{l}\mathbf{L} \\
\mathbf{E}\end{array}$ \\
\hline Sub-1 & $\mathrm{S}$ & S & $\mathrm{S}$ & $\mathrm{S}$ & I & $\mathrm{R}$ & S & S & $\mathrm{R}$ & $\mathrm{R}$ & $\mathrm{S}$ & $\mathrm{R}$ & $\mathrm{R}$ & $\mathrm{R}$ & 1 & $\mathrm{R}$ & $\mathrm{R}$ & $\mathrm{S}$ & $\mathrm{R}$ & $\mathrm{R}$ & $\mathrm{R}$ & 1 & $\mathrm{R}$ & $\mathrm{R}$ & $\mathrm{S}$ & $\mathrm{R}$ & $\mathrm{R}$ & $\mathrm{R}$ \\
\hline Sub-2 & $\mathrm{S}$ & S & S & $\mathrm{S}$ & I & $\mathrm{R}$ & $\mathrm{S}$ & $S$ & $\mathrm{R}$ & $\mathrm{R}$ & $\mathrm{S}$ & $\mathrm{R}$ & $\mathrm{R}$ & $\mathrm{R}$ & I & $\mathrm{R}$ & $\mathrm{R}$ & $\mathrm{S}$ & $\mathrm{R}$ & $\mathrm{R}$ & $\mathrm{R}$ & $\mathrm{S}$ & $\mathrm{R}$ & $\mathrm{R}$ & $\mathrm{S}$ & $\mathrm{R}$ & $\mathrm{R}$ & $\mathrm{R}$ \\
\hline Sub-3 & $\mathrm{S}$ & S & $\mathrm{S}$ & $\mathrm{S}$ & S & $\mathrm{R}$ & S & S & $\mathrm{R}$ & $\mathrm{R}$ & $\mathrm{S}$ & $\mathrm{R}$ & $\mathrm{R}$ & $\mathrm{R}$ & $S$ & $\mathrm{R}$ & $\mathrm{R}$ & $\mathrm{S}$ & $\mathrm{R}$ & $\mathrm{R}$ & $\mathrm{R}$ & $\mathrm{S}$ & $\mathrm{R}$ & $\mathrm{R}$ & $\mathrm{S}$ & $\mathrm{R}$ & $\mathrm{R}$ & $\mathrm{R}$ \\
\hline Sub-4 & $\mathrm{S}$ & S & $\mathrm{S}$ & $\mathrm{S}$ & S & $\mathrm{R}$ & S & S & $\mathrm{R}$ & $\mathrm{R}$ & $\mathrm{S}$ & $\mathrm{R}$ & $\mathrm{R}$ & $\mathrm{R}$ & $\pi$ & $\mathrm{R}$ & $\mathrm{R}$ & $\mathrm{S}$ & $\mathrm{R}$ & $\mathrm{R}$ & $\mathrm{R}$ & S & $\mathrm{R}$ & $\mathrm{R}$ & $\mathrm{S}$ & $\mathrm{R}$ & $\mathrm{R}$ & $\mathrm{R}$ \\
\hline Sub-5 & $\mathrm{S}$ & $\mathrm{S}$ & $\mathrm{S}$ & $\mathrm{S}$ & S & I & $\mathrm{S}$ & $\mathrm{S}$ & $\mathrm{R}$ & $\mathrm{R}$ & $\mathrm{S}$ & $\mathrm{R}$ & $\mathrm{R}$ & $\mathrm{R}$ & $S$ & $\mathrm{R}$ & $\mathrm{R}$ & $\mathrm{S}$ & $\mathrm{R}$ & $\mathrm{R}$ & $\mathrm{R}$ & S & $\mathrm{R}$ & $\mathrm{R}$ & $\mathrm{S}$ & $\mathrm{R}$ & $\mathrm{R}$ & $\mathrm{R}$ \\
\hline
\end{tabular}




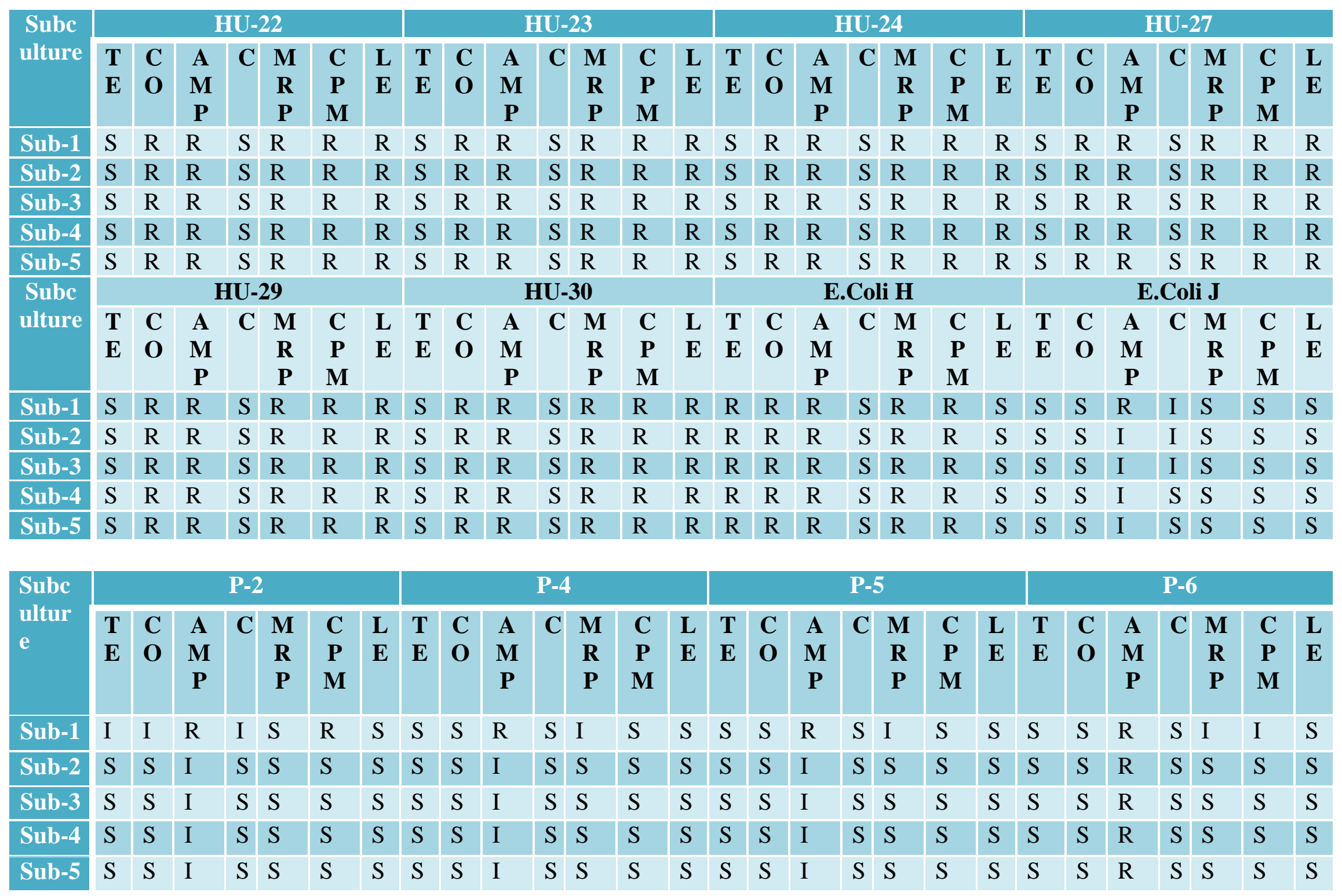




\begin{tabular}{|c|c|c|c|c|c|c|c|c|c|c|c|c|c|c|c|c|c|c|c|c|c|c|c|c|c|c|c|c|}
\hline \multirow{2}{*}{$\begin{array}{c}\text { Subc } \\
\text { ulture }\end{array}$} & \multicolumn{7}{|c|}{ P-9 } & \multicolumn{7}{|c|}{ P-10 } & \multicolumn{7}{|c|}{ P-12 } & \multicolumn{7}{|c|}{ P-15 } \\
\hline & $\begin{array}{l}\mathbf{T} \\
\mathbf{E}\end{array}$ & $\begin{array}{l}\mathbf{C} \\
\mathbf{O}\end{array}$ & $\begin{array}{c}\mathbf{A} \\
\mathbf{M} \\
\mathbf{P}\end{array}$ & C & $\begin{array}{l}\mathbf{M} \\
\mathbf{R} \\
\mathbf{P}\end{array}$ & $\begin{array}{l}\mathbf{C} \\
\mathbf{P} \\
\mathbf{M}\end{array}$ & $\begin{array}{l}\mathbf{L} \\
\mathbf{E}\end{array}$ & $\begin{array}{l}\mathbf{T} \\
\mathbf{E}\end{array}$ & $\begin{array}{l}\mathbf{C} \\
\mathbf{O}\end{array}$ & $\begin{array}{l}\mathbf{A} \\
\mathbf{M} \\
\mathbf{P}\end{array}$ & C & $\begin{array}{l}\mathbf{M} \\
\mathbf{R} \\
\mathbf{P}\end{array}$ & $\begin{array}{l}\mathbf{C} \\
\mathbf{P} \\
\mathbf{M}\end{array}$ & $\begin{array}{l}\mathbf{L} \\
\mathbf{E}\end{array}$ & $\begin{array}{l}\mathbf{T} \\
\mathbf{E}\end{array}$ & $\begin{array}{l}\mathbf{C} \\
\mathbf{O}\end{array}$ & $\begin{array}{l}\mathbf{A} \\
\mathbf{M} \\
\mathbf{P}\end{array}$ & C & $\begin{array}{l}\mathbf{M} \\
\mathbf{R} \\
\mathbf{P}\end{array}$ & $\begin{array}{l}\mathbf{C} \\
\mathbf{P} \\
\mathbf{M}\end{array}$ & $\begin{array}{l}\mathbf{L} \\
\mathbf{E}\end{array}$ & $\begin{array}{l}\mathbf{T} \\
\mathbf{E}\end{array}$ & $\begin{array}{l}\text { C } \\
\mathbf{O}\end{array}$ & $\begin{array}{l}\mathbf{A} \\
\mathbf{M} \\
\mathbf{P}\end{array}$ & & $\begin{array}{l}\mathbf{M} \\
\mathbf{R} \\
\mathbf{P}\end{array}$ & $\begin{array}{l}\mathbf{C} \\
\mathbf{P} \\
\mathbf{M}\end{array}$ & $\begin{array}{l}\mathbf{L} \\
\mathbf{E}\end{array}$ \\
\hline Sub-1 & S & $\mathrm{S}$ & $\mathrm{R}$ & $S$ & I & $\mathrm{S}$ & $\mathrm{S}$ & I & $\mathrm{S}$ & $\mathrm{R}$ & $\mathrm{S}$ & $\mathrm{R}$ & $\mathrm{R}$ & $\mathrm{S}$ & S & $\mathrm{S}$ & $\mathrm{R}$ & $S$ & I & $\mathrm{S}$ & $\mathrm{S}$ & $\mathrm{S}$ & S & $\mathrm{R}$ & $S s$ & $S$ & $\mathrm{~S}$ & $S$ \\
\hline Sub-2 & $\mathrm{S}$ & S & $\mathrm{R}$ & S & I & $\mathrm{S}$ & $\mathrm{S}$ & $\mathrm{S}$ & $\mathrm{S}$ & I & $\mathrm{S}$ & S & $\mathrm{S}$ & $\mathrm{S}$ & S & S & I & $\mathrm{S}$ & I & S & $\mathrm{S}$ & $\mathrm{S}$ & S & I & $S s$ & S & $\mathrm{S}$ & $S$ \\
\hline Sub-3 & S & $S$ & $\mathrm{R}$ & $S$ & $\mathrm{~S}$ & $S$ & $\mathrm{~S}$ & $S$ & $\mathrm{~S}$ & I & $S$ & $\mathrm{~S}$ & $S$ & $\mathrm{~S}$ & S & $\mathrm{S}$ & I & $S$ & $S$ & $S$ & $\mathrm{~S}$ & $\mathrm{~S}$ & S & I & $S s$ & $S$ & $\mathrm{~S}$ & $S$ \\
\hline Sub-4 & S & S & $\mathrm{R}$ & S & S & $S$ & S & $\mathrm{S}$ & S & I & $\mathrm{S}$ & $\mathrm{S}$ & $S$ & $\mathrm{~S}$ & S & $\mathrm{S}$ & I & $\mathrm{S}$ & S & $\mathrm{S}$ & $\mathrm{S}$ & $\mathrm{S}$ & S & S & $S s$ & S & S & $S$ \\
\hline Sub-5 & S & $\mathrm{S}$ & $\mathrm{R}$ & $S$ & $\mathrm{~S}$ & $S$ & $S$ & $S$ & $\mathrm{~S}$ & I & $S$ & $\mathrm{~S}$ & $S$ & $\mathrm{~S}$ & $S$ & $\mathrm{~S}$ & I & S & $\mathrm{S}$ & $\mathrm{S}$ & $\mathrm{S}$ & $\mathrm{S}$ & $\mathrm{S}$ & S & $S s$ & S & $\mathrm{S}$ & $S$ \\
\hline \multirow{2}{*}{$\begin{array}{l}\text { Subc } \\
\text { ulture }\end{array}$} & \multicolumn{7}{|c|}{ P-16 } & \multicolumn{7}{|c|}{ P-19 } & \multicolumn{7}{|c|}{ P-21 } & \multicolumn{7}{|c|}{ P-22 } \\
\hline & $\begin{array}{l}\mathbf{T} \\
\mathbf{E}\end{array}$ & $\begin{array}{l}\text { C } \\
\text { O }\end{array}$ & $\begin{array}{l}\mathbf{A} \\
\mathbf{M} \\
\mathbf{P}\end{array}$ & C & $\begin{array}{l}\mathbf{M} \\
\mathbf{R} \\
\mathbf{P}\end{array}$ & $\begin{array}{l}\mathbf{C} \\
\mathbf{P} \\
\mathbf{M}\end{array}$ & $\begin{array}{l}\mathbf{L} \\
\mathbf{E}\end{array}$ & $\begin{array}{l}\mathbf{T} \\
\mathbf{E}\end{array}$ & $\begin{array}{l}\text { C } \\
\text { O }\end{array}$ & $\begin{array}{l}\mathbf{A} \\
\mathbf{M} \\
\mathbf{P}\end{array}$ & C & $\begin{array}{l}\mathbf{M} \\
\mathbf{R} \\
\mathbf{P}\end{array}$ & $\begin{array}{l}\mathbf{C} \\
\mathbf{P} \\
\mathbf{M}\end{array}$ & $\begin{array}{l}\mathbf{L} \\
\mathbf{E}\end{array}$ & $\begin{array}{l}\mathbf{T} \\
\mathbf{E}\end{array}$ & $\begin{array}{l}\text { C } \\
\mathbf{O}\end{array}$ & $\begin{array}{l}\mathbf{A} \\
\mathbf{M} \\
\mathbf{P}\end{array}$ & C & $\begin{array}{l}\mathbf{M} \\
\mathbf{R} \\
\mathbf{P}\end{array}$ & $\begin{array}{l}\mathbf{C} \\
\mathbf{P} \\
\mathbf{M}\end{array}$ & $\begin{array}{l}\mathbf{L} \\
\mathbf{E}\end{array}$ & $\begin{array}{l}\mathbf{T} \\
\mathbf{E}\end{array}$ & $\begin{array}{l}\mathbf{C} \\
\mathbf{O}\end{array}$ & $\begin{array}{c}\mathbf{A} \\
\mathbf{M} \\
\mathbf{P}\end{array}$ & C & $\begin{array}{l}\mathbf{M} \\
\mathbf{R} \\
\mathbf{P}\end{array}$ & $\begin{array}{l}\mathbf{C} \\
\mathbf{P} \\
\mathbf{M}\end{array}$ & $\begin{array}{l}\mathbf{L} \\
\mathrm{E}\end{array}$ \\
\hline Sub-1 & S & S & I & S & S & $\mathrm{S}$ & S & $\mathrm{S}$ & $\mathrm{S}$ & $\mathrm{R}$ & S & $\mathrm{R}$ & $\mathrm{S}$ & S & S & $\mathrm{S}$ & I & S & $S$ & $\mathrm{~S}$ & $\mathrm{~S}$ & I & $S$ & $\mathrm{R}$ & S: & S & $\mathrm{S}$ & \\
\hline Sub-2 & S & S & I & $S$ & S & S & S & S & S & $\mathrm{R}$ & $S$ & S & S & S & S & S & I & $S$ & S & S & S & S & S & $\mathrm{R}$ & $\mathrm{S}:$ & S & S & $S$ \\
\hline Sub-3 & S & S & I & $S$ & S & S & S & S & S & $\mathrm{R}$ & S & S & $\mathrm{S}$ & $\mathrm{S}$ & S & S & I & $S$ & S & S & S & S & S & $\mathrm{R}$ & $S$ & $S$ & S & \\
\hline Sub-4 & S & S & I & $\mathrm{S}$ & S & S & S & S & S & $\mathrm{R}$ & S & S & S & S & S & S & I & $S$ & S & S & S & S & S & $\mathrm{R}$ & S : & S & S & $S$ \\
\hline Sub-5 & S & S & I & S & S & $S$ & S & S & $\mathrm{S}$ & $\mathrm{R}$ & $S$ & S & $\mathrm{S}$ & $\mathrm{S}$ & $S$ & $\mathrm{~S}$ & I & S & $S$ & $\mathrm{~S}$ & S & $\mathrm{S}$ & S & $\mathrm{R}$ & $\mathrm{S}:$ & S & S & $S$ \\
\hline \multirow{2}{*}{$\begin{array}{l}\text { Subc } \\
\text { ultur } \\
\text { e }\end{array}$} & \multicolumn{7}{|c|}{ PouF-2 } & \multicolumn{7}{|c|}{ PouF-3 } & \multicolumn{7}{|c|}{ PouF-4 } & \multicolumn{7}{|c|}{ PouS-9 } \\
\hline & $\begin{array}{l}\mathbf{T} \\
\mathbf{E}\end{array}$ & $\begin{array}{l}\mathbf{C} \\
\mathbf{O}\end{array}$ & $\begin{array}{l}\mathbf{A} \\
\mathbf{M} \\
\mathbf{P}\end{array}$ & C & $\begin{array}{l}\mathbf{M} \\
\mathbf{R} \\
\mathbf{P}\end{array}$ & $\begin{array}{l}\mathbf{C} \\
\mathbf{P} \\
\mathbf{M}\end{array}$ & $\begin{array}{l}\mathbf{L} \\
\mathbf{E}\end{array}$ & $\begin{array}{l}\mathbf{T} \\
\mathbf{E}\end{array}$ & $\begin{array}{l}\mathbf{C} \\
\mathbf{O}\end{array}$ & $\begin{array}{l}\mathbf{A} \\
\mathbf{M} \\
\mathbf{P}\end{array}$ & C & $\begin{array}{l}\mathbf{M} \\
\mathbf{R} \\
\mathbf{P}\end{array}$ & $\begin{array}{l}\mathbf{C} \\
\mathbf{P} \\
\mathbf{M}\end{array}$ & $\begin{array}{l}\text { L } \\
\text { E }\end{array}$ & $\begin{array}{l}\mathbf{T} \\
\mathbf{E}\end{array}$ & $\begin{array}{l}\text { C } \\
\mathbf{O}\end{array}$ & $\begin{array}{c}\mathbf{A} \\
\mathbf{M} \\
\mathbf{P}\end{array}$ & C & $\begin{array}{l}\mathbf{M} \\
\mathbf{R} \\
\mathbf{P}\end{array}$ & $\begin{array}{l}\mathbf{C} \\
\mathbf{P} \\
\mathbf{M}\end{array}$ & $\mathbf{L}$ & $\begin{array}{l}\mathbf{T} \\
\mathbf{E}\end{array}$ & $\begin{array}{l}\text { C } \\
\mathbf{O}\end{array}$ & $\begin{array}{c}\mathbf{A} \\
\mathbf{M} \\
\mathbf{P}\end{array}$ & C & $\begin{array}{l}\mathbf{M} \\
\mathbf{R} \\
\mathbf{P}\end{array}$ & $\begin{array}{l}\mathbf{C} \\
\mathbf{P} \\
\mathbf{M}\end{array}$ & $\begin{array}{l}\mathrm{L} \\
\mathrm{E}\end{array}$ \\
\hline Sub-1 & $\mathrm{R}$ & S & $\mathrm{R}$ & $\mathrm{S}$ & I & $\mathrm{R}$ & S & $\mathrm{R}$ & $\mathrm{R}$ & $\mathrm{R}$ & $\mathrm{S}$ & I & $\mathrm{R}$ & S & $\mathrm{R}$ & S & $\mathrm{R}$ & $\begin{array}{ll}S & \text { I }\end{array}$ & I & $\mathrm{R}$ & S & $\mathrm{R}$ & S & I & $\mathrm{S}$ & S & $S$ & $S$ \\
\hline Sub-2 & $\mathrm{R}$ & $S$ & $\mathrm{R}$ & S & I & $\mathrm{R}$ & $S$ & $\mathrm{R}$ & $\mathrm{R}$ & $\mathrm{R}$ & S & I & $\mathrm{R}$ & $S$ & $\mathrm{R}$ & $S$ & $\mathrm{R}$ & \begin{tabular}{l|l}
$S$ & I
\end{tabular} & I & I & $S$ & $\mathrm{R}$ & $S$ & I & $\mathrm{S}$ & $S$ & $S$ & S \\
\hline Sub-3 & $\mathrm{R}$ & S & $\mathrm{R}$ & $\mathrm{S}$ & I & $\mathrm{R}$ & $S$ & $\mathrm{R}$ & $\mathrm{R}$ & I & $\mathrm{S}$ & I & $\mathrm{R}$ & S & $\mathrm{R}$ & S & S & $\begin{array}{lll}S & \text { I }\end{array}$ & I & I & S & $\mathrm{R}$ & S & I & $\mathrm{S}$ & $S$ & $S$ & $S$ \\
\hline Sub-4 & $\mathrm{R}$ & S & $\mathrm{R}$ & S & I & $\mathrm{R}$ & S & $\mathrm{R}$ & $\mathrm{R}$ & I & S & I & $\mathrm{R}$ & S & $\mathrm{R}$ & S & S & \begin{tabular}{l|l}
$S$ & I
\end{tabular} & I & I & S & $\mathrm{R}$ & S & I & S & S & S & \\
\hline Sub-5 & $\mathrm{R}$ & S & $\mathrm{R}$ & $\mathrm{S}$ & I & $\mathrm{R}$ & S & $\mathrm{R}$ & $\mathrm{R}$ & I & S & I & $\mathrm{R}$ & S & $\mathrm{R}$ & S & S & $\begin{array}{ll}S & \text { I }\end{array}$ & I & I & S & $\mathrm{R}$ & S & I & S & S & S & S \\
\hline
\end{tabular}




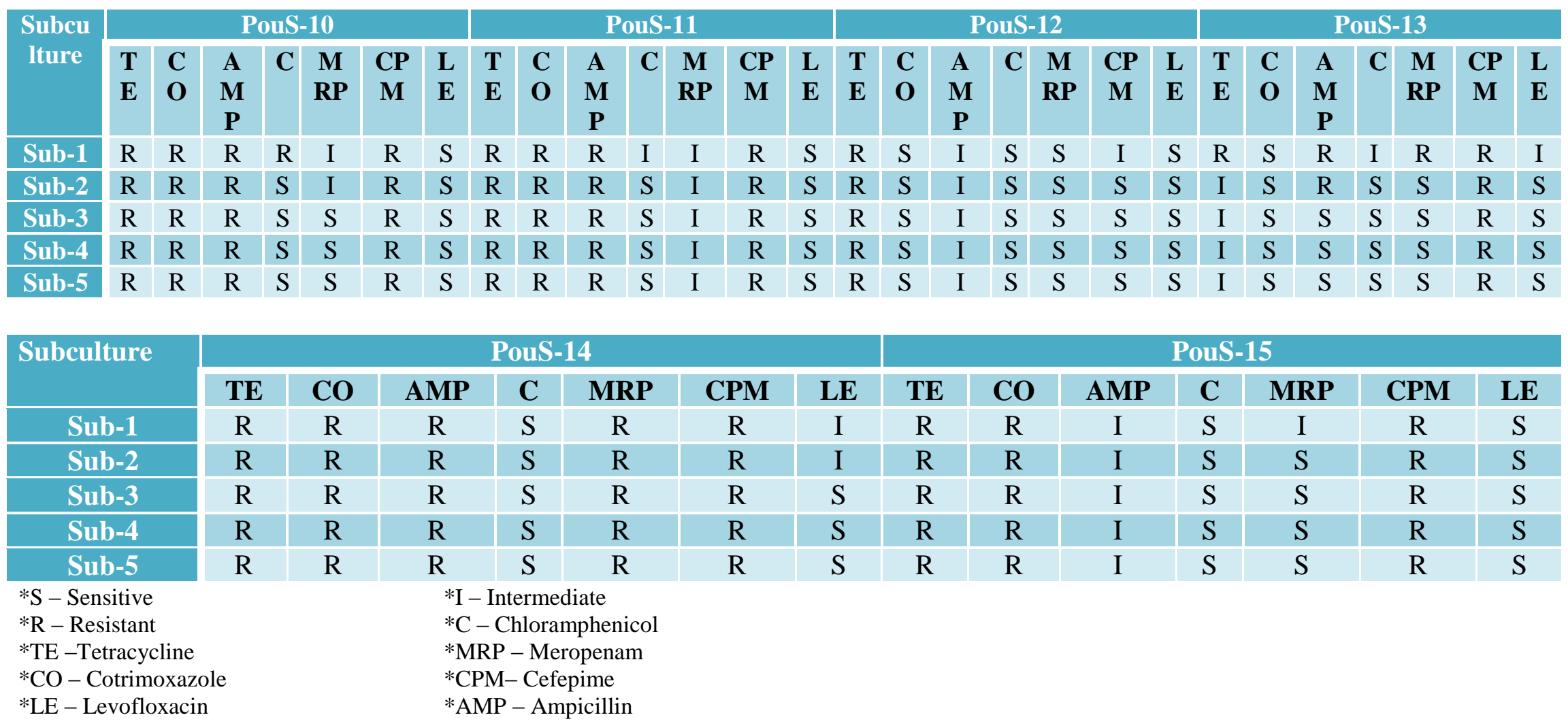

Table.7 Number of $E$. coli isolate showing decrease in resistance on different sub culturing against tested group of antibiotics

\begin{tabular}{|c|c|c|c|c|c|c|}
\hline S. No & Name of antibiotics & $\begin{array}{l}\text { No. of isolates ( } 2^{\text {nd }} \\
\text { sub culture) }\end{array}$ & $\begin{array}{l}\text { No. of isolates ( } 3^{\text {rd }} \\
\text { sub culture) }\end{array}$ & $\begin{array}{l}\text { No. of isolates ( } 4^{\text {th }} \\
\text { sub culture) }\end{array}$ & $\begin{array}{l}\text { No. of isolates }\left(5^{\text {th }}\right. \\
\text { sub culture) }\end{array}$ & Total isolate \\
\hline 1. & Meropenam & 11 & 5 & 1 & 0 & 17 \\
\hline 2. & Ampicillin & 6 & 7 & 1 & 0 & 14 \\
\hline 3. & Chloramphenicol & 7 & 0 & 1 & 0 & 8 \\
\hline 4. & Cefepime & 6 & 0 & 0 & 1 & 7 \\
\hline 5. & Tetracycline & 6 & 1 & 0 & 0 & 7 \\
\hline 6. & Levofloxacin & 4 & 1 & 1 & 0 & 6 \\
\hline 7. & Co-trimoxazole & 2 & 0 & 0 & 0 & 2 \\
\hline
\end{tabular}


Table.8 Intensity of florescence of different E. coli isolates at different concentrations of Et-br after $1^{\text {st }}$ sub culturing

\begin{tabular}{|c|c|c|}
\hline Intensity & Isolates not showing fluorescence & Isolates showing fluorescence \\
\hline \multicolumn{3}{|c|}{ Result of florescence at $0.5 \mathrm{mg} / \mathrm{l}$ concentration } \\
\hline Human & $\begin{array}{l}\text { HU2, HU4, HU7, HU17, HU18, HU20, HU22, HU23, } \\
\text { HU24, HU27, HU29, HU30, E.H. }\end{array}$ & HU3, HS10, HU12,HU13, HU14, HU15, E.J. \\
\hline Pig & $\mathrm{P} 2, \mathrm{P} 4, \mathrm{P} 10, \mathrm{P} 12, \mathrm{P} 15$ & P5, P6, P9, P16, P19, P21, P22 \\
\hline Poultry & PouS9, PouS12 & PouF2, PouF3, PouF4, PouS10, PouS11, PouS13, PouS14, PouS15 \\
\hline \multicolumn{3}{|c|}{ Result of florescence at $1 \mathrm{mg} / \mathrm{l}$ concentration } \\
\hline Human & $\begin{array}{l}\text { HU2, HU7, HU17, HU18, HU20, HU22, HU23, HU24, } \\
\text { HU27, HU29, HU30, E.H. }\end{array}$ & HU3, HU4, HS10, HU12, HU13, HU14, HU15, E.J. \\
\hline Pig & $\mathrm{P} 2, \mathrm{P} 4, \mathrm{P} 10, \mathrm{P} 12, \mathrm{P} 15$ & P5, P6, P9, P16, P19, P21, P22 \\
\hline Poultry & PouS9, PouS12 & PouF2, PouF3, PouF4, PouS10, PouS11, PouS13, PouS14, PouS15 \\
\hline \multicolumn{3}{|c|}{ Result of florescence at $1.5 \mathrm{mg} / \mathrm{l}$ concentration } \\
\hline Human & HU17, HU18, HU22, HU23, HU24, HU27, HU29, HU30 & $\begin{array}{l}\text { HU2, HU3, HU4, HU7, HS10, HU12, HU13, HU14, HU15, HU20, } \\
\text { E.J., E.H. }\end{array}$ \\
\hline Pig & $\mathrm{P} 2, \mathrm{P} 4, \mathrm{P} 12, \mathrm{P} 15$ & P5, P6, P9, P10, P16, P19, P21, P22 \\
\hline Poultry & PouS9, PouS12 & PouF2, PouF3, PouF4, PouS10, PouS11, PouS13, PouS14, PouS15 \\
\hline \multicolumn{3}{|c|}{ Result of florescence at $2 \mathrm{mg} / \mathrm{l}$ concentration } \\
\hline Human & HU17, HU18, HU22, HU23, HU24, HU27, HU29, HU30 & $\begin{array}{l}\text { HU2, HU3, HU4, HU7, HS10, HU12, HU13, HU14, HU15, HU20, } \\
\text { E.J., E.H. }\end{array}$ \\
\hline Pig & P12, P15 & $\mathrm{P} 2, \mathrm{P} 4, \mathrm{P} 5, \mathrm{P} 6, \mathrm{P} 9, \mathrm{P} 10, \mathrm{P} 16, \mathrm{P} 19, \mathrm{P} 21, \mathrm{P} 22$ \\
\hline Poultry & PouS12 & $\begin{array}{l}\text { PouF2, PouF3, PouF4, PouS9, PouS10, PouS11, PouS13, PouS14, } \\
\text { PouS15 }\end{array}$ \\
\hline \multicolumn{3}{|c|}{ Result of florescence at $2.5 \mathrm{mg} / \mathrm{l}$ concentration } \\
\hline Human & HU17, HU18, HU22, HU23, HU24, HU27, HU29, HU30 & $\begin{array}{l}\text { HU2, HU3, HU4, HU7, HS10, HU12, HU13, HU14, HU15, HU20, } \\
\text { E.J., E.H. }\end{array}$ \\
\hline Pig & NIL & P2, P4, P5, P6, P9, P10, P12, P15, P16, P19, P21, P22 \\
\hline Poultry & NIL & $\begin{array}{l}\text { PouF2, PouF3, PouF4, PouS9, PouS10, PouS11, PouS12, PouS13, } \\
\text { PouS14, PouS15 }\end{array}$ \\
\hline
\end{tabular}


Table.9 Intensity of florescence of different $E$. coli isolates at different concentrations of Et-br after $2^{\text {nd }}$ sub culturing

\begin{tabular}{|c|c|c|}
\hline Intensity & Isolates not showing fluorescence & Isolates showing fluorescence \\
\hline \multicolumn{3}{|c|}{ Result of florescence at $\mathbf{0 . 5} \mathrm{mg} / \mathrm{l}$ concentration } \\
\hline Human & $\begin{array}{l}\text { HU2, HU4, HU17, HU18, HU20, HU22, HU23, HU24, } \\
\text { HU27, HU29, HU30, E.H. }\end{array}$ & HU3, HU7, HS10, HU12, HU13, HU14, HU15, E.J, E.H. \\
\hline Pig & $\mathrm{P} 2, \mathrm{P} 4, \mathrm{P} 10, \mathrm{P} 12, \mathrm{P} 15$ & P5, P6, P9, P16, P19, P21, P22 \\
\hline Poultry & PouS9, PouS12 & PouF2, PouF3, PouF4, PouS10, PouS11, PouS13, PouS14, PouS15 \\
\hline \multicolumn{3}{|c|}{ Result of florescence at $1 \mathrm{mg} / \mathrm{l}$ concentration } \\
\hline Human & $\begin{array}{l}\text { HU2, HU17, HU20, HU22, HU23, HU24, HU27, HU29, } \\
\text { HU30, }\end{array}$ & $\begin{array}{l}\text { HU3, HU4, HU7, HS10, HU12, HU13, HU14, HU15, HU18, E.J., } \\
\text { E.H. }\end{array}$ \\
\hline Pig & NIL & $\mathrm{P} 2, \mathrm{P} 4, \mathrm{P} 5, \mathrm{P} 6, \mathrm{P} 9, \mathrm{P} 10, \mathrm{P} 12, \mathrm{P} 15, \mathrm{P} 16, \mathrm{P} 19, \mathrm{P} 21, \mathrm{P} 22$ \\
\hline Poultry & PouS9, PouS12 & PouF2, PouF3, PouF4, PouS10, PouS11, PouS13, PouS14, PouS15 \\
\hline \multicolumn{3}{|c|}{ Result of florescence at $1.5 \mathrm{mg} / \mathrm{l}$ concentration } \\
\hline Human & HU27, HU29, HU30 & $\begin{array}{l}\text { HU2, HU3, HU4, HU7, HS10, HU12, HU13, HU14, HU15, HU20, } \\
\text { HU17, HU18, HU22, HU23, HU24, E.J., E.H. }\end{array}$ \\
\hline Pig & NIL & P2, P4, P5, P6, P9, P10, P12, P15, P16, P19, P21, P22 \\
\hline Poultry & PouS9, PouS12 & PouF2, PouF3, PouF4, PouS10, PouS11, PouS13, PouS14, PouS15 \\
\hline \multicolumn{3}{|c|}{ Result of florescence at $2 \mathrm{mg} / \mathrm{l}$ concentration } \\
\hline Human & NIL & $\begin{array}{l}\text { HU2, HU3, HU4, HU7, HS10, HU12, HU13, HU14, HU15, HU20, } \\
\text { HU17, HU18, HU22, HU23, HU24, HU27, HU29, HU30, E.J., E.H. }\end{array}$ \\
\hline Pig & NIL & P2, P4, P5, P6, P9, P10, P12, P15, P16, P19, P21, P22 \\
\hline Poultry & PouS12 & $\begin{array}{l}\text { PouF2, PouF3, PouF4, PouS9, PouS10, PouS11, PouS13, PouS14, } \\
\text { PouS15 }\end{array}$ \\
\hline \multicolumn{3}{|c|}{ Result of florescence at $2.5 \mathrm{mg} / \mathrm{l}$ concentration } \\
\hline Human & NIL & $\begin{array}{l}\text { HU2, HU3, HU4, HU7, HS10, HU12, HU13, HU14, HU15, HU20, } \\
\text { HU17, HU18, HU22, HU23, HU24, HU27, HU29, HU30, E.J., E.H. }\end{array}$ \\
\hline Pig & NIL & P2, P4, P5, P6, P9, P10, P12, P15, P16, P19, P21, P22 \\
\hline Poultry & NIL & $\begin{array}{l}\text { PouF2, PouF3, PouF4, PouS9, PouS10, PouS11, PouS12, PouS13, } \\
\text { PouS14, PouS15 }\end{array}$ \\
\hline
\end{tabular}


Table.10 Intensity of florescence of different E. coli isolates at different concentrations of Et-br after $3^{\text {rd }}$ sub culturing

\begin{tabular}{|c|c|c|}
\hline Intensity & Isolates not showing fluorescence & Isolates showing fluorescence \\
\hline \multicolumn{3}{|c|}{ Result of florescence at $0.5 \mathrm{mg} / \mathrm{l}$ concentration } \\
\hline Human & $\begin{array}{l}\text { HU2, HU17, HU18, HU20, HU22, HU23, HU24, } \\
\text { HU27, HU29, HU30, E.H. }\end{array}$ & HU3, HU4, HU7, HS10, HU12, HU13, HU14, HU15, E.J, E.H. \\
\hline Pig & $\mathrm{P} 2, \mathrm{P} 4, \mathrm{P} 10, \mathrm{P} 12, \mathrm{P} 15$ & P5, P6, P9, P16, P19, P21, P22 \\
\hline Poultry & PouS9, PouS12 & PouF2, PouF3, PouF4, PouS10, PouS11, PouS13, PouS14, PouS15 \\
\hline \multicolumn{3}{|c|}{ Result of florescence at $1 \mathrm{mg} / \mathrm{l}$ concentration } \\
\hline Human & HU17, HU22, HU23, HU24, HU27, HU29, HU30, & $\begin{array}{l}\text { HU2, HU3, HU4, HU7, HS10, HU12, HU13, HU14, HU15, HU18, } \\
\text { HU20, E.J., E.H. }\end{array}$ \\
\hline Pig & NIL & $\mathrm{P} 2, \mathrm{P} 4, \mathrm{P} 5, \mathrm{P} 6, \mathrm{P} 9, \mathrm{P} 10, \mathrm{P} 12, \mathrm{P} 15, \mathrm{P} 16, \mathrm{P} 19, \mathrm{P} 21, \mathrm{P} 22$ \\
\hline Poultry & PouS9, PouS12 & PouF2, PouF3, PouF4, PouS10, PouS11, PouS13, PouS14, PouS15 \\
\hline \multicolumn{3}{|c|}{ Result of florescence at $1.5 \mathrm{mg} / \mathrm{l}$ concentration } \\
\hline Human & HU27, HU29, HU30 & $\begin{array}{l}\text { HU2, HU3, HU4, HU7, HS10, HU12, HU13, HU14, HU15, HU20, } \\
\text { HU17, HU18, HU22, HU23, HU24, E.J., E.H. }\end{array}$ \\
\hline Pig & NIL & P2, P4, P5, P6, P9, P10, P12, P15, P16, P19, P21, P22 \\
\hline Poultry & PouS9, PouS12 & PouF2, PouF3, PouF4, PouS10, PouS11, PouS13, PouS14, PouS15 \\
\hline \multicolumn{3}{|c|}{ Result of florescence at $2 \mathrm{mg} / \mathrm{l}$ concentration } \\
\hline Human & NIL & $\begin{array}{l}\text { HU2, HU3, HU4, HU7, HS10, HU12, HU13, HU14, HU15, HU20, } \\
\text { HU17, HU18, HU22, HU23, HU24, HU27, HU29, HU30, E.J., E.H. }\end{array}$ \\
\hline Pig & NIL & P2, P4, P5, P6, P9, P10, P12, P15, P16, P19, P21, P22 \\
\hline Poultry & PouS12 & $\begin{array}{l}\text { PouF2, PouF3, PouF4, PouS9, PouS10, PouS11, PouS13, PouS14, } \\
\text { PouS15 }\end{array}$ \\
\hline \multicolumn{3}{|c|}{ Result of florescence at $2.5 \mathrm{mg} / \mathrm{l}$ concentration } \\
\hline Human & NIL & $\begin{array}{l}\text { HU2, HU3, HU4, HU7, HS10, HU12, HU13, HU14, HU15, HU20, } \\
\text { HU17, HU18, HU22, HU23, HU24, HU27, HU29, HU30, E.J., E.H. }\end{array}$ \\
\hline Pig & NIL & P2, P4, P5, P6, P9, P10, P12, P15, P16, P19, P21, P22 \\
\hline Poultry & NIL & $\begin{array}{l}\text { PouF2, PouF3, PouF4, PouS9, PouS10, PouS11, PouS12, PouS13, } \\
\text { PouS14, PouS15 }\end{array}$ \\
\hline
\end{tabular}


Table.11 Intensity of florescence of different E. coli isolates at different concentrations of Et-br after $4^{\text {th }}$ sub culturing

\begin{tabular}{|c|c|c|}
\hline Intensity & Isolates not showing fluorescence & Isolates showing fluorescence \\
\hline \multicolumn{3}{|c|}{ Result of florescence at $0.5 \mathrm{mg} / \mathrm{l}$ concentration } \\
\hline Human & $\begin{array}{l}\text { HU17, HU18, HU20, HU22, HU23, HU24, HU27, HU29, } \\
\text { HU30, E.H. }\end{array}$ & $\begin{array}{l}\text { HU2, HU3, HU4, HU7, HS10, HU12, HU13, HU14, HU15, E.J, } \\
\text { E.H. }\end{array}$ \\
\hline Pig & $\mathrm{P} 2, \mathrm{P} 4, \mathrm{P} 10, \mathrm{P} 12, \mathrm{P} 15$ & P5, P6, P9, P16, P19, P21, P22 \\
\hline Poultry & PouS9, PouS12 & PouF2, PouF3, PouF4, PouS10, PouS11, PouS13, PouS14, PouS15 \\
\hline \multicolumn{3}{|c|}{ Result of florescence at $1 \mathrm{mg} / \mathrm{l}$ concentration } \\
\hline Human & HU17, HU22, HU23, HU24, HU27, HU29, HU30, & $\begin{array}{l}\text { HU2, HU3, HU4, HU7, HS10, HU12, HU13, HU14, HU15, HU18, } \\
\text { HU20, E.J., E.H. }\end{array}$ \\
\hline Pig & NIL & P2, P4, P5, P6, P9, P10, P12, P15, P16, P19, P21, P22 \\
\hline Poultry & PouS9, PouS12 & PouF2, PouF3, PouF4, PouS10, PouS11, PouS13, PouS14, PouS15 \\
\hline \multicolumn{3}{|c|}{ Result of florescence at $1.5 \mathrm{mg} / \mathrm{l}$ concentration } \\
\hline Human & NIL & $\begin{array}{l}\text { HU2, HU3, HU4, HU7, HS10, HU12, HU13, HU14, HU15, HU20, } \\
\text { HU17, HU18, HU22, HU23, HU24, HU27, HU29, HU30, E.J., E.H. }\end{array}$ \\
\hline Pig & NIL & P2, P4, P5, P6, P9, P10, P12, P15, P16, P19, P21, P22 \\
\hline Poultry & PouS9, PouS12 & PouF2, PouF3, PouF4, PouS10, PouS11, PouS13, PouS14, PouS15 \\
\hline \multicolumn{3}{|c|}{ Result of florescence at $2 \mathrm{mg} / \mathrm{l}$ concentration } \\
\hline Human & NIL & $\begin{array}{l}\text { HU2, HU3, HU4, HU7, HS10, HU12, HU13, HU14, HU15, HU20, } \\
\text { HU17, HU18, HU22, HU23, HU24, HU27, HU29, HU30, E.J., E.H. }\end{array}$ \\
\hline Pig & NIL & P2, P4, P5, P6, P9, P10, P12, P15, P16, P19, P21, P22 \\
\hline Poultry & PouS12 & $\begin{array}{l}\text { PouF2, PouF3, PouF4, PouS9, PouS10, PouS11, PouS13, PouS14, } \\
\text { PouS15 }\end{array}$ \\
\hline \multicolumn{3}{|c|}{ Result of florescence at $2.5 \mathrm{mg} / \mathrm{l}$ concentration } \\
\hline Human & NIL & $\begin{array}{l}\text { HU2, HU3, HU4, HU7, HS10, HU12, HU13, HU14, HU15, HU20, } \\
\text { HU17, HU18, HU22, HU23, HU24, HU27, HU29, HU30, E.J., E.H. }\end{array}$ \\
\hline Pig & NIL & P2, P4, P5, P6, P9, P10, P12, P15, P16, P19, P21, P22 \\
\hline Poultry & NIL & $\begin{array}{l}\text { PouF2, PouF3, PouF4, PouS9, PouS10, PouS11, PouS12, PouS13, } \\
\text { PouS14, PouS15 }\end{array}$ \\
\hline
\end{tabular}


Table.12 Intensity of florescence of different $E$. coli isolates at different concentrations of Et-br after $5^{\text {th }}$ sub culturing

\begin{tabular}{|c|c|c|}
\hline Intensity & Isolates not showing fluorescence & Isolates showing fluorescence \\
\hline \multicolumn{3}{|c|}{ Result of florescence at $0.5 \mathrm{mg} / \mathrm{l}$ concentration } \\
\hline Human & HU17, HU18, HU20, HU23, HU24, HU30, E.H. & $\begin{array}{l}\text { HU2, HU3, HU4, HU7, HS10, HU12, HU13, HU14, HU15, HU22, } \\
\text { HU27, HU29, E.J, E.H. }\end{array}$ \\
\hline Pig & $\mathrm{P} 2, \mathrm{P} 4, \mathrm{P} 10, \mathrm{P} 12, \mathrm{P} 15$ & P5, P6, P9, P16, P19, P21, P22 \\
\hline Poultry & PouS9, PouS12 & PouF2, PouF3, PouF4, PouS10, PouS11, PouS13, PouS14, PouS15 \\
\hline \multicolumn{3}{|c|}{ Result of florescence at $1 \mathrm{mg} / \mathrm{l}$ concentration } \\
\hline Human & NIL & $\begin{array}{l}\text { HU2, HU3, HU4, HU7, HS10, HU12, HU13, HU14, HU15, } \\
\text { HU117, HU18, HU20, HU22, HU23, HU24, HU27, HU29, HU30, } \\
\text { E.J., E.H. }\end{array}$ \\
\hline Pig & NIL & $\mathrm{P} 2, \mathrm{P} 4, \mathrm{P} 5, \mathrm{P} 6, \mathrm{P} 9, \mathrm{P} 10, \mathrm{P} 12, \mathrm{P} 15, \mathrm{P} 16, \mathrm{P} 19, \mathrm{P} 21, \mathrm{P} 22$ \\
\hline Poultry & PouS9, PouS12 & PouF2, PouF3, PouF4, PouS10, PouS11, PouS13, PouS14, PouS15 \\
\hline \multicolumn{3}{|c|}{ Result of florescence at $1.5 \mathrm{mg} / \mathrm{l}$ concentration } \\
\hline Human & NIL & $\begin{array}{l}\text { HU2, HU3, HU4, HU7, HS10, HU12, HU13, HU14, HU15, HU20, } \\
\text { HU17, HU18, HU22, HU23, HU24, HU27, HU29, HU30, E.J., E.H. }\end{array}$ \\
\hline Pig & NIL & P2, P4, P5, P6, P9, P10, P12, P15, P16, P19, P21, P22 \\
\hline Poultry & PouS9, PouS12 & PouF2, PouF3, PouF4, PouS10, PouS11, PouS13, PouS14, PouS15 \\
\hline \multicolumn{3}{|c|}{ Result of florescence at $2 \mathrm{mg} / \mathrm{l}$ concentration } \\
\hline Human & NIL & $\begin{array}{l}\text { HU2, HU3, HU4, HU7, HS10, HU12, HU13, HU14, HU15, HU20, } \\
\text { HU17, HU18, HU22, HU23, HU24, HU27, HU29, HU30, E.J., E.H. }\end{array}$ \\
\hline Pig & NIL & P2, P4, P5, P6, P9, P10, P12, P15, P16, P19, P21, P22 \\
\hline Poultry & PouS12 & $\begin{array}{l}\text { PouF2, PouF3, PouF4, PouS9, PouS10, PouS11, PouS13, PouS14, } \\
\text { PouS15 }\end{array}$ \\
\hline \multicolumn{3}{|c|}{ Result of florescence at $2.5 \mathrm{mg} / \mathrm{l}$ concentration } \\
\hline Human & NIL & $\begin{array}{l}\text { HU2, HU3, HU4, HU7, HS10, HU12, HU13, HU14, HU15, HU20, } \\
\text { HU17, HU18, HU22, HU23, HU24, HU27, HU29, HU30, E.J., E.H. }\end{array}$ \\
\hline Pig & NIL & P2, P4, P5, P6, P9, P10, P12, P15, P16, P19, P21, P22 \\
\hline Poultry & NIL & $\begin{array}{l}\text { PouF2, PouF3, PouF4, PouS9, PouS10, PouS11, PouS12, PouS13, } \\
\text { PouS14, PouS15 }\end{array}$ \\
\hline
\end{tabular}


The product of these loci then function as a multidrug efflux pump with broad substrate specificity or, in the case of the mar locus, as a regulator to control expression of at least one membrane efflux system, $a c r A B$ (Ross et al., 1998).

All the members of Enterobacteriaceae are intrinsically resistant (100\%) to RNA inhibitor Rifampicin as the RNA polymerase of E. coli is 1000 fold less sensitive to Rifampicin as compared to that of $M$. tuberculosis (Zenkin et al., 2005). This may be the reason that all isolates were resistant to Rifampicin. For Trimethoprim $65 \%$ of human and poultry isolates were resistant. Although, initially Trimethoprim was effective against $E$. coli and was effectively used in the treatments of Urinary Tract Infections caused by $E$. coli but its extensive use selected for resistant strains. In $E$. coli, trimethoprimsulfamethoxazole resistance often correlates with the presence of dihydrofolate reductase (DHFR) and dihydropteroate synthase (DHPS) genes in integrons (Rasheed et al., 2014). Even though there has been drastic decrease in the use of trimethoprim but the low fitness cost of trimethoprim resistance and its ease of spread with the integrons has made least effect on its resistance (Brolund et al., 2010). All the pig isolates were sensitive to this antibiotic.

The fluoroquinolone antibiotics were considered to be critical antibiotics due to their very high effectiveness against pathogens that are resistant to other antibiotics (Olorunmola et al., 2013). In present study for the quinolone group of antibiotics (DNA synthesis inhibitors) 61.9\%, $33.3 \%$ and $26.2 \%$ isolates were resistant for Nalidixic acid, Ciprofloxacin and Levofloxacin respectively (Table 2). In an earlier study, the strains with intermediate resistance to ciprofloxacin were reported to have a single mutation in gyrA or gyrA and
parC genes, and strains with complete resistance to ciprofloxacin had double and single mutations in gyrA and parC, respectively (Guerra et al., 2003). In the present study it was also found that there were low levels of resistance (13.6\% and $4.5 \%$ ) in pig and poultry (30\% and 10\%) isolates for Ciprofloxacin and Levofloxacin as compared to human isolates $(55 \%$ and $50 \%)$. Quinolones are drug of choice for the urinary tract infections (UTI). Since there were isolates of $E$. coli from patients with UTI it could be the probable reason for resistance towards this group of antibiotics.

\section{Ethidium bromide agar (Et-br) cartwheel test based evaluation of MDR strains}

The results of Ethidium bromide agar (Et-br) cartwheel method for evaluation of MDR is given in Table 5. At a concentration of 0.5 $\mathrm{mg} / \mathrm{l}, 81 \%$ of the isolates resisted the entry of Et-br. But at the higher concentration of Et-br i.e. $1 \mathrm{mg} / \mathrm{l}$ to $2.5 \mathrm{mg} / \mathrm{l}$ there was uptake of Etbr in a dose dependent manner and more isolates started to show florescence. But, there were ten isolates i.e. HU-17, HU-18, HU-22, HU-23, HU-24, HU-27, HU-29, HU-30, PouF-9 and PouF-12 which could resist the uptake of Et-br even at a dose of $2.5 \mathrm{mg} / \mathrm{l}$.

This test is a subjective test where exclusion of the Et-br by the bacterial isolates leads to no florescence and vice-versa. The extent of exclusion is indicative of the capability of an isolate to prevent the entry of antibiotics. In this approach, higher the concentration of Etbr required to produce fluorescence of the bacterial mass, greater is the capacity of the bacterial cells to resist higher concentrations of antibiotics also. This test can be used as an assessment test for methods of resistance in MDR phenotype bacteria. The prevention of the entry may be because of the active efflux pump activity due to which the Et-br is actively pumped out of the bacterial cell or it 
may be because of the decrease in the number of porins which otherwise are the sites of drug entry in a bacterial isolate (Martins et al., 2013). Based on the above results it could be deduced that the above ten isolates had higher capacity to prevent the entry of antibiotics.

\section{Evaluation of MDR $E$. coli isolates for adaptive resistance}

All the $42 E$. coli isolates were sub-cultured five times to see whether the withdrawal of antibiotics had any effect on the resistivity of the isolates and permeability of Et-br and at which level. To study resistivity patterns seven antibiotics were taken namely: Tetracycline, Chloramphenicol, Ampicillin, Cefepime, Meropenum, Levofloxacin and Cotrimoxazole. In majority of the isolates positive effect of withdrawal of antibiotic was observed. The isolates became sensitive to an antibiotic either on second or third subculturing but not on first sub-culture. No significant change in pattern was observed post third sub culturing. But, the extent of sensitivity varied for different antibiotics on the isolates irrespective of the source of the isolates i.e. human, pig or poultry. All the isolates behaved as an independent identity. To deduce a uniform pattern, so that overall effect of a given antibiotic could be studied the results of each isolate were evaluated against a given antibiotic. Effect of subculturing was observed maximum for Meropenum where a total of 17 isolates showed the positive effect in terms of increased sensitivity followed by Ampicillin (Table 6 and 7). It could be deduced that the withdrawal of antibiotic does have a positive effect on the resistance of bacteria. In a study to investigate the temporal occurrence and the genetic background of apramycin and gentamicin resistance in Escherichia coli strains from pork, duration of use and amounts of antibiotics used; both had a significant effect on the prevalence of apramycin/gentamicin cross-resistance (Jensen et al., 2006). Yet another study on influence of prophylactic and therapeutic antimicrobial administration on antimicrobial resistance of faecal $E$. coli in a dairy calves Berge et al., 2006 reported transient effect of antibiotic on resistance.

To further assess whether the permeability of the isolates increased on sub-culturing in an antibiotic free environment, isolates were cultured in presence of Et-br. Sub-culturing had a positive effect on the permeability of Et-br as indicated by the fluorescence given by the bacteria post each sub culture (Table 8 to 12). The isolates initially resisting higher doses of Et-br on subsequent culturing became more permeable to higher doses of Et-br. Initially, there were ten isolates that were resisting high dose $(2.5 \mathrm{mg} / \mathrm{l})$ of Et-br. But by fifth sub-culturing none of the isolate was resisting the high dose $(2.5 \mathrm{mg} / \mathrm{l})$ of Etbr. There was only one isolate (PouS 12) which could resist dose of $2 \mathrm{mg} / \mathrm{l}$ on fifth subculturing. This clearly indicates that withdrawal of antibiotics had a drastic positive effect on the susceptibility of bacteria where almost all the isolates became permeable to even $1 \mathrm{mg} / \mathrm{l}$ dose of Et-br.

Reductions in prescribing antimicrobials have been reported to achieve specific, localized decreases in antimicrobial resistance (Barbosa and Levy, 2000). In Japan, erythromycin resistance decreased from $22.2 \%$ of clinical isolates to virtually $100 \%$ sensitivity over a 9year-period following reduced macrolide consumption (Barbosa and Levy, 2000). Reduction of resistance following the removal of antimicrobial treatment is reported by Nys et al., (2005) reported reduction of resistance following removal of antibiotic treatment. But, on the contrary, some studies have illustrated that resistance can persist over a prolonged period to antimicrobial agents no longer in common use (Enne et al., 2001). 
It depends on several factors whether the withdrawal of antibiotics will always have a positive effect. Therefore, from the present study, it can only be stated that under in vitro conditions there was a generalized positive effect of withdrawal on the permeability of isolates to Et-br but resistivity patterns indicate that this withdrawal has a selective effect.

Effect of sub-culturing in antibiotic free environment lead to positive effect on resistivity pattern, although it varied for different antibiotic for different isolates but the permeability of all the isolates increased uniformly to Et-br. It was concluded that each isolate has an independent identity and can adopt any mechanism present in it to resist the antibiotic toxicity but withdrawal of antibiotic helps in reverting the resistant phenotype towards being susceptible in majority of the MDRs under in vitro conditions.

\section{Acknowledgement}

I am thankful to Co-authors Prof. Sunil Maherchandani, Prof. S. K. Kashyap Department of Veterinary Microbiology \& Biotechnology, Bikaner for his guidance, cooperation and persistent motivation during entire period of study.

\section{References}

Barbosa, T.M. and Levy, S.B. (2000). The impact of antibiotic use on resistance development and persistence. Drug Res. Updates 3: 303-311.

Barclay, M.L. and Begg, E.J. (2001). Aminoglycoside adaptive resistance: importance for effective dosage regimens. Drugs. 61: 713-721.

Berge, A.C.B., Moore, D.A. and Sischo, W.M. (2006). Field Trial Evaluating the Influence of Prophylactic and Therapeutic Antimicrobial Administration on Antimicrobial Resistance of Fecal
Escherichia coli in Dairy Calves. Appl. Environ. Microbiol. 72(6): 3872-3878.

Brolund, A., Sundqvist, M., Kahlmeter, G. and Grape, M. (2010). Molecular Characterization of Trimethoprim Resistance in Escherichia coli and Klebsiella pneumoniae during a Two Year Intervention on Trimethoprim Use. PLoS ONE. 5(2): 9233.

Courvalin, P. (1994). Transfer of antibiotic resistance genes between grampositive and gram-negative bacteria. Antimicrob. Agents Chemother. 38: 1447-1451.

Enne, V.I., Livermore, D.M., Stephens, P. and Hall, L.M. (2001). Persistence of sulphonamide resistance in Escherichia coli in the UK despite national prescribing restriction. Lancet. 357: 1325-1328.

Ganguly, N.K., Arora, N.K., Chandy, S.J., Fairoze, M.N., Gill, J.P.S., Gupta, U., Hossain, S., Joglekar, S., Joshi, P. C., Kakkar, M., Kotwani, A., Rattan, A., Sudarshan, H., Thomas, K., Wattal, C., Easton, A. and Laxminarayan, R. (2011). Rationalizing antibiotic use to limit antibiotic resistance in India. J. Med. Res. 134(3): 281-294.

Guerra, B., Junker, E., Schroeter, A., Malorny, B., Lehmann, S. and Helmuth, R. (2003). Phenotypic and genotypic characterization of antimicrobial resistance in German Escherichia coli isolates from cattle, swine and poultry. $J$. Antimicrob. Chemother. 52(3): 489-492.

Jensen, V.F., Jakobsen, L., Emborg, H., Seyfarth, A.M. and Hammerum, A.M. (2006). Correlation between apramycin and Gentamicin use in pigs and an increase reservoir of Gentamicin-resistant Escherichia coli. J. Antimicrob. Chemother. 58(1): 101.

Laxminarayan, R. and Chaudhury, R.R. (2016). Antibiotic Resistance in India: Drivers and Opportunities for Action. PLoS Med. 13(3): e1001974.

Little, M.L., Qin, X., Zerr, D.M. and Weissman, S.J. (2012). Molecular diversity in mechanisms of carbapenems resistance in 
paediatric Enterobacteriaceae. Int. J. Antimicrob. Agents. 39(1): 52-57.

Magnet, S. and Blanchard, J.S. (2005). Molecular insights into aminoglycosides action and resistance. Chem. Rev. 105: 477-497.

Martins, M., McCusker, M.P., Viveiros, M., Couto, I., Fanning, S., Pagès, J.M., and Amaral, L. (2013). A Simple Method for Assessment of MDR Bacteria for OverExpressed Efflux Pumps. Open Microbiol. J. 7(1): 72-82.

Meena, H. (2015). Mechanism of antibiotic resistance in multidrug resistant commensal Escherichia coli isolated from poultry. M. V. Sc. Thesis submitted to the Rajasthan University of Veterinary and Animal Science, Bikaner.

Nys, S., Bruinsma, N., Filius, P.M.G., van den Bogaard, A.E., Hoffman, L., Terporten, P.H.W., Wildeboer-Veloo, A.C.M., Degener, J., Endtz, H.P. and Stobberingh, E.E. (2005). Effect of hospitalisation on the antibiotic resistance of faecal Enterococcus faecalis of surgical patients over time. Microbial Drug Resis. 11: 154-158.

Olorunmola, F.O., Kolawole, D.O. and Lamikanra, A. (2013). Antibiotic Resistance and Virulence Properties in Escherichia coli Strains from Cases of Urinary Tract Infections. Afr. J. Infect. Dis. 7(1): 1-7.

Quinn, P.J., Carter, M.E., Markey, B.K. and Carter, G.R. (1994). Clin. Vet. Microbiol. Wolf Publishing, Mosby-Year Book Europe Ltd. Lynton House, 7-12. Tavistock Square, London WCH 9LB, England.
Rasheed, M.U., Thajuddin, N., Ahamed, P., Teklemariam, Z. and Jamil, K. (2014). Antimicrobial drug resistance in strains of Escherichia coli isolated from food sources. Rev. Inst. Med. Trop. Sao Paulo. 56(4): 341-346.

Robinson, T.P., Bu, D.P., Carrique-Mas, J., Fevre, E.M., Gilbert, M., Grace, D., Hay, S.I., Jiwakanon, J., Kakkar, M., Kariuki, S., Laxminarayan, R., Lubroth, J., Magnusson, U., Thi Ngoc, P., Van Boeckel, T.P. and Woolhouse, M.E.J. (2016). Antibiotic resistance is the quintessential One Health issue. Trans. R. Soc. Trop. Med. Hyg. 110(7): 377-380.

Ross, J.I., Eady, E.A., Cove, J.H., and Cunliffe, W.J. (1998). 16S rRNA Mutation Associated with Tetracycline Resistance in a Gram-Positive Bacterium. Antimicrob. Agents Chemother. 42(7): 1702-1705.

Van Boeckel, T.P., Gandra, S., Ashok, A., Caudron, Q., Grenfell, B.T., Levin, S.A. and Laxminarayan, R. (2014). Global antibiotic consumption 2000 to 2010: an analysis of national pharmaceutical sales data. Lancet Infect. Dis. 14(8): 742-750.

Walsh, C.T., Fisher, S.L., Park, I.S., Prahalad, M. and $\mathrm{Wu}, \mathrm{Z}$. (1996). Bacterial resistance to vancomycin: five genes and one missing hydrogen bond tell the story. Chemistry \& Biology. 3: 21-28.

Zenkin, N., Kulbachinskiy, A., Bass, I. and Nikiforov, V. (2005). Different Rifampin Sensitivities of Escherichia coli and Mycobacterium tuberculosis RNA Polymerases are not explained by the difference in the subunit Rifampin regions I and II. Antimicrob. Agents Chemother. 49(4): 1587-1590.

\section{How to cite this article:}

Hitesh Kumar and Sunil Maherchandani. 2018. Effects of Antibiotic Withdrawal on Multidrug Resistant Escherichia coli. Int.J.Curr.Microbiol.App.Sci. 7(10): 161-182. doi: https://doi.org/10.20546/ijcmas.2018.710.018 